\title{
Analytical criteria to quantify and compare the antioxidant and pro-oxidant capacity in competition assays: The bell protection function.
}

\author{
M A. Prieto ${ }^{1,2}$, M. A. Murado ${ }^{2}$, J. A. Vázquez ${ }^{2} \&$ Thomas P. Curran $^{1}$ \\ ${ }^{1}$ UCD School of Biosystems Engineering, University College Dublin, Belfield, Dublin 4, \\ Ireland. \\ ${ }^{2}$ Research Marine Institute (IIM-CSIC), Group of Recycling and Valorization of Waste \\ Materials (REVAL), Vigo, Spain.
}

\begin{abstract}
The development of a convenient mathematical application for testing the antioxidant and prooxidant potential of standard and novel therapeutic agents is essential for the research community and food industry in order to perform more precise evaluations of products and processes. In this work, a simple non-linear dose-time tool to test the effectiveness of compounds for competitive assays is presented. The model helps to describe accurately the antioxidant and pro-oxidant response as a function of time and dose by two criteria values and allows one to perform easily comparisons of both capacities from different compounds. The quantification procedure developed was applied to two well known in vitro competition assays, the $\beta$-carotene and crocin bleaching asymptotic reactions. The dose-time dependency of the response of commercial antioxidants and some expected pro-oxidant compounds was evaluated in this study and the results showed low experimental error. In addition, as an illustrative example of the capabilities of the criteria proposed, the quantification of the combined effect of an antioxidant and a pro-oxidant was analyzed. Afterwards, the model was verified for other relevant competitive methods, using available experimental data from the bibliography. Its application is simple, it provides parametric estimates which characterize the response, and it facilitates rigorous comparisons among the effects of different compounds and experimental approaches. In all experimental data tested, the calculated parameters were always statistically significant (Student's t-test, $\alpha=0.05$ ), the equations were consistent (Fisher's F-test) and the goodness of fit coefficient of determination was higher than 0.98 .
\end{abstract}

Keywords: antioxidant activity; pro-oxidant activity; competition methods; mathematical modeling; time-dose response. 


\section{INTRODUCTION}

Antioxidants and pro-oxidants are compounds that can delay or accelerate oxidation processes. Living organisms have developed a complex network (Kalyanaraman, 2004) of antioxidants (enzymes such as superoxide dismutase, catalase, glutathione peroxidase or non-enzymatic compounds such as uric acid, bilirubin, albumin, metallothioneins); they are essential for a healthy life in order to counteract various harmful (Hussain et al., 2003) pro-oxidants or reactive species (i.e. $\mathrm{O}_{2}, \mathrm{H}_{2} \mathrm{O}_{2}, \mathrm{ROO}^{\bullet}, \mathrm{OH}^{\bullet}$ ). Apart from these endogenous antioxidants, there are exogenous ones that can derive from natural sources (vitamins, flavonoids, anthocyanins, some mineral compounds), or from synthetic compounds (such as butylhydroxyanisole, butylhydroxytoluene, etc). There are also exogenous compounds such as metal ions that can promote or accelerate the oxidation processes (Carocho \& Ferreira, 2013). Clinical trials and epidemiological studies have established an inverse correlation between the intake of natural exogenous antioxidants and the occurrence of oxidative stress diseases such as inflammation, cardiovascular problems, cancer, and aging-related disorders (Gutteridge \& Halliwell, 2010). Thus, the analysis of natural antioxidants for disease prevention (Chatterjee et al., 2005; Notas et al., 2005) and the identification of possible pro-oxidant substances have become topics of increasing interest.

Several in vivo and in vitro methods have been developed for determining the total antioxidant and pro-oxidant (oxidation modifiers, OM) capacity of compounds. The capacity of OM is frequently determined in competition assays, in which the $\mathrm{OM}$ and indicators of the reaction (in general another OM) compete for the reactive species. Competition assays are performed to describe OM capacity and to rank the affinity of OM to counteract or increase the action of reactive species against an indicator. In general, these assays differ in the mechanism of generation of different radical species and/or target molecules and in the way end-products are measured. At present, there is no convenient assay that enables the evaluation of the OM capacity (Naguib, 2000; Tsuchihashi et al., \& Niki, 1995; Halliwell, 2013) for different compounds. The current methods to test the OM capacity still have left many open questions (Frankel \& Meyer, 2000; Halliwell, 2012). The in vitro assays can only rank OM capacity for their particular reaction system and their relevance to in vivo activities is uncertain. Thus, it is logical that in the last decade, researchers have claimed unity of the approaches (Frankel \& Finley, 2008; Murado \& Vázquez, 2010) and have tended to standardize the protocols to increase the effectiveness of methods for in vitro and in vivo responses (Dawidowicz \& Olszowy, 2010; Frankel, 1993; 1994; Ordoudi \& Tsimidou, 2006; Prior et al., 2005; 1999).

Additionally, the arbitrary use of simple analytical procedures to calculate molecular properties, occasionally without a validation study, as well as a lack of statistical significance, has caused much controversy (Frankel, 1993; 1994; Huang et al., 2005; Koleva et al., 2002; Laguerre \& Villeneuve, 2007; Naguib, 2000; Roginsky, 2005). Commonly, the mathematical determinations of the OM capacity are based on a fixed endpoint without proper considerations of the kinetic behavior. The most typical and incorrect practice is to use the single-time dose-response of one commercial $\mathrm{OM}$ as a calibration curve (normally focusing on the linear range), and afterwards to compute the equivalent OM capacity of any type of sample by testing it only at one single-timedose, assuming too many false aspects as true.

In the current study, a simple non-linear mathematical application for competitive OM assays, in which the responses have one common asymptote (majority of ones) is presented. It helps to describe accurately the response as a function of time and dose by two criteria values and facilitates convenient comparisons of the capacity of different compounds. The model was 
validated in well known in vitro competition assays, evaluating the dose-time-dependency of the response of OM compounds.

\section{MATERIAL AND METHODS}

\section{1. $\beta$-carotene bleaching method}

The protocol has been recently revised and improved (Prieto et al., 2012). The reagent is prepared by dissolving $4 \mathrm{mg}$ of $\beta$-carotene $(\beta \mathrm{C}), 0.5 \mathrm{~mL}$ of linoleic acid and $4 \mathrm{~g}$ of Tween-40 in $20 \mathrm{~mL}$ of chloroform. In aliquots of $1 \mathrm{~mL}$, the solution was distributed into $30 \mathrm{~mL}$ tubes, and the chloroform was evaporated simultaneously in all of them in a rotary evaporator $\left(40{ }^{\circ} \mathrm{C} / \sim 15 \mathrm{~min}\right)$, adapted to work with multiple tubes. The resulting oily residue was washed with $\mathrm{N}_{2}$ and stored at $-18{ }^{\circ} \mathrm{C}$. At the time of use, a tube provides sufficient reagents for 120 samples by adding 30 $\mathrm{mL}$ of buffer Briton $100 \mathrm{mM}, \mathrm{pH}=6.5$ in Mili-Q water at the reaction temperature $\left(45^{\circ} \mathrm{C}\right)$. The absorbance at $470 \mathrm{~nm}$ of the reagent thus prepared is $\sim 1.4$, stable for a week and the specific value should not be corrected for dilution. The concentration of $\beta C$ in the final solution of the reaction is $1 \mu \mathrm{M}$.

The procedure is performed by adding $50 \mu \mathrm{L}$ of sample and $250 \mu \mathrm{L}$ of reagent into the wells $(330 \mathrm{~mL})$ of a microplate of 96 units (it is advisable to use a multichannel pipette). The device is programmed to $45^{\circ} \mathrm{C}$ with agitation for reading only interrupting at intervals of 3,5 and 10 minutes (initiation, propagation and asymptotic phase), during a period of 200 minutes. The $O M$ standards and samples are analyzed kinetically for different doses. Under these conditions the method can be applied to analyze antioxidants and pro-oxidants separately or even simultaneously.

\subsection{Crocin bleaching assay}

Recently, the protocol has been revised and its quantification procedure improved and transfered to microplate readers (Prieto et al., 2013a; 2013b) The reagent is prepared by dissolving $\mathrm{Cr}$ (5 $\mathrm{mg} ; 125 \mu \mathrm{M}$ in the final reaction) and AAPH (75 mg; $7.68 \mathrm{mM}$ in the final reaction) in 25 and 5 $\mathrm{mL}$, respectively, of $100 \mathrm{mM}$ Briton buffer, $\mathrm{pH}=5.5$, in Mili-Q water at $40{ }^{\circ} \mathrm{C}$. To avoid any initial degradation, both solutions must be prepared and mixed just before use. The absorbance at $450 \mathrm{~nm}$ of the mixture $(\sim 1.4)$ is very dependent on the origin and conservation state of Cr. The concentration of $\mathrm{Cr}$ in the final solution of the reaction is $100 \mu \mathrm{M}$. When applying the method to analyze pro-oxidants the AAPH compound must not be included in the reagent preparation, all other conditions are maintained.

Each well of a preheated $\left(37^{\circ} \mathrm{C}\right)$ microplate $(96$ wells, $350 \mu \mathrm{l})$ contains $250 \mu \mathrm{l}$ of reagent, $50 \mu \mathrm{l}$ of sample solution in water:ethanol (9:1). The apparatus was programmed for $200 \mathrm{~min}$ at $37{ }^{\circ} \mathrm{C}$, with agitation at 660 cycles/min ( $1 \mathrm{~mm}$ amplitude), only interrupted for readings at intervals of 3, 5 and 10 min (initiation, propagation and asymptotic phase).

\subsection{Standard OM compounds for an illustrative analysis}

\subsubsection{Antioxidants}

(a) Butyl-hydroxyanisole (BHA): a synthetic food additive (E320) mainly used as an antioxidant and preservative. Its known capacity is suitable in lipophilic and hydrophilic environments. 
(b) Butyl-hydroxytoluene (BHT): a synthetic lipophilic (fat-soluble) organic compound, chemically a derivative of phenol, that is useful for its antioxidant properties. It is primarily used as a food additive (E321).

(c) Propyl 3,4,5-trihydroxybenzoate or propyl gallate (PG): an antioxidant that has been added to foods containing oils and fats to prevent oxidation (E310).

(d) (2R)-2,5,7,8-tetramethyl-2-[(4R,8R)-(4,8,12-trimethyltridecyl)]-6-chromanol or $\alpha$ tocopherol (TOC): a natural fat-soluble organic compound (E306) consisting of various methylated phenols (a type of tocopherol or vitamin E), that is useful for its antioxidant properties.

(e) 6-ethoxy-2,2,4-trimethyl-1,2-dihydroquinoline or ethoxyquin (ETX): commonly used as a food preservative (E324) in pet foods to prevent the rancidification of fats, in spices to prevent color loss due to oxidation of the natural carotenoid pigments and as a pesticide.

(f) L-hexuronic acid (vitamin C) or Ascorbic Acid (AA): a naturally occurring hydrosoluble organic compound with antioxidant properties. Ascorbic acid and its sodium, potassium, and calcium salts are commonly used as antioxidant food additives (E300-304)

(g) Tert-Butylhydroquinone (TBHQ): It is a derivative of hydroquinone, substituted with tert-butyl group. TBHQ is a highly effective antioxidant in foods (E319). It is added to a wide range of foods, with the highest limit $(1000 \mathrm{mg} / \mathrm{kg})$ permitted for frozen fish and fish products.

(h) Manganese sulfate $\left(\mathrm{Mn}^{+2}\right)$ : a required trace mineral for all known living organisms, also extensively present as possible interference in salts may be able to act as a metal chelator (e.g., iron-sequestrants) and inhibit Fenton-type reactions that produce hydroxyl radicals through complexation/chelation reactions.

(i) 6-hydroxy-2,5,7,8-tetramethylchroman-2-carboxylic acid (Trolox, Tr): A water-soluble analog of vitamin $\mathrm{E}$ used in biological or biochemical applications to reduce oxidative stress or damage.

The concentration ranges in $\mu \mathrm{M}$ of the antioxidants used for the $\beta \mathrm{C}$ reaction are: BHA: 0-(0.5)-5, BHT: 0-(3)-30, ETX: 0-(0.0004)-0.004, TOC: 0-(0.004)-0.04, PG: 0-(8)-80. The concentration ranges in $\mu \mathrm{M}$ of the antioxidants used for the Cr reaction are: AA: 0-(30)-300, ETX: 0-(3)-30, Tr: 0-(15)-150, TBHQ: 0-(80)-800, $\mathrm{Mn}^{+2}$ : 0-(12.5)-125. All compounds were purchased from Sigma S.A. (St. Louis, MO, USA).

\subsubsection{Potential pro-oxidant agents}

(a) Iron (II) sulfide $\left(\mathrm{Fe}^{+2}\right)$ : much attention has been paid to its oxygen complexes (ferryl and perferryl radical) in the food industry as they are considered as primary catalysts (initiators) of lipid peroxidation in meat products and others that contain lipids.

(b) Porcine Hemoglobin $(\mathrm{Hb})$ in reduced form $\left(\mathrm{Fe}^{+2}\right)$ : the iron-containing oxygen-transport metalloprotein in the red blood cells. Hb can be found in many food compounds interfering with its antioxidant activity and also is a typical compound that caused rapid rancidity.

(c) Copper (II) sulfate $\left(\mathrm{Cu}^{+2}\right)$ : an essential trace nutrient to all higher plant and animal life, also widely present in biological extracts, water and as possible interference in salts.

(d) AAPH (2,2'-Azobis(2-amidinopropane) dihydrochloride): a hydrophilic chemical compound used to study the chemistry of the oxidation of drugs or the capabilities of antioxidants in different.

The concentration ranges in $\mu \mathrm{M}$ of the antioxidants used for the $\beta \mathrm{C}$ reaction are: $\mathrm{Fe}^{+2} 0-(1.5)-15$; $\mathrm{Cu}^{+2}$ 0-(15)-240; $\mathrm{Hb}$ 0-(2)-20.0. For the $\mathrm{Cr}$ reaction AAPH 0-(12.5)-125 was used. All compounds were purchased from Sigma S.A. (St. Louis, MO, USA).

\subsection{Numerical and statistical methods}


Fitting the experimental results to the proposed equations was carried out in two phases. First, parametric estimates were obtained by minimization of the sum of quadratic differences between observed and model-predicted values, using the nonlinear least-square (quasi-Newton) method provided by the macro Solver in Microsoft Excel 2003, which allows quick testing of hypotheses and display of its consequences. Next, the determination of the parametric confidence intervals and model consistency (Student's $t$ and Fisher's $F$ tests, respectively, in both cases with $\alpha=0.05$ ) were calculated using the 'SolverAid' (Prikler, 2009). The 'SolverStat' macro (Comuzzi et al., 2003; Prieto et al, 2011) was used for detecting possible anomalies in the distribution of parametric estimates and residuals.

\section{RESULTS}

At first, as an example, experimental data values are used to illustrate the capabilities of the method, and afterwards, the quantification and comparative method was applied to different combinations of $\mathrm{OM}$ compounds in two competition assays (the $\beta \mathrm{C}$ and $\mathrm{Cr}$ bleaching reactions). Then, to illustrate its capabilities, the model was further extended to the analysis of the combine effect of an antioxidant and a pro-oxidant simultaneously. Finally, some methods in which the quantification and comparative method of the OM capacity could be potentially applied, are presented, and data from other authors was used to extend the validation of the procedure into another competitive assays.

\subsection{Illustration of the bell protection function and simple analytical criteria to compare the time-dose response of compounds}

In competitive assays, performed in systems without limitations of oxygen, it can be accepted that exhaustive substrate oxidation is reached at sufficient time, and therefore the final asymptotic value will be equal for all the kinetic responses in absence and presence of any type of OM. The method developed here can only be applied if this requirement is fulfilled, which is the case of the most common competitive assays in the oxidation field.

Data obtained in the $\beta C$ bleaching reaction is used to illustrate the procedure to assess the capacity of OM. The antioxidant of BHT and the pro-oxidant $\mathrm{Fe}^{+2}$ as a function of time and dose are used as example.

\subsubsection{Standardizations and fittings}

The first step is to standardize the response, thus all kinetic profiles in the presence of a concentration of an OM are subtracted by the kinetic profile in its absence, as follows:

$$
R D(t)=O M_{t}-C_{t}
$$

in which $O M$ and $C$ are the kinetic response in the presence and absence (control) of an oxidation modifier concentration, respectively. $R D$ is the relative difference found at any given time $(t)$, which in this case accounts for the amount of $\mu \mathrm{M}$ of $\beta \mathrm{C}$ or $\mathrm{Cr}$ protected by the $\mathrm{OM}$ agent. When the agent is a pro-oxidant the profile will be a negative bell function and when it is an antioxidant will be a positive bell profile.

In Figure 1, a representation of the characteristic profiles obtained by $\beta C$ bleaching reaction using a time-dose response of the antioxidant of BHT and the pro-oxidant $\mathrm{Fe}^{+2}$ is presented. 
Observing the response (Figure 1, top), it is clear that the analysis of this profile, with simple mathematical relations measured at one single time, will produce under- or over-estimations of the capacity of the antioxidant, depending on the time selected. Alternatively, the traditional option is the analysis with S-shaped equations, producing several parameters that characterize the response of the remaining $\beta C$ molecules through the lag-time period, the time required for reaching half maximum response, the maximum bleaching rate, etc. However, our proposal, the kinetic relative difference response, exhibits an asymmetric bell profile (Figure 1, bottom), which is equivalent to the substrate molecules protected (positive for antioxidants and negative for pro-oxidants) by the OM molecules as a function of time. Such profiles show many different physical kinetic properties that could characterize the response. Among these physical properties, the maximum protected molecules of $\beta \mathrm{C}\left(P_{\mathrm{m}}\right)$ and the time at which it takes place $\left(t_{\mathrm{m}}\right)$ are the most characterizing parameters that cannot be found through traditional equations. For example, in the food industry, the combinatory use of these parameters could provide the state of the oxidation of the reaction after the chain reaction will be inevitably affecting the taste, flavors and other properties of foods, because it focuses on analyzing the quantity of protection and the moment at which such protection would be lost.

This characteristic bell protection profile can be described by many bell functions (Di Marco, 2001). After testing several equations, the generalized exponential function without intercept (also called the modified Weibull distribution function) was found to be the most satisfactory one with least number of parameters and highest accuracy:

$$
R D(t)=P_{m}\left\{\frac{i}{d}\left[1-\left(\frac{t}{t_{m}}\right)^{d}+\ln \left(\frac{t}{t_{m}}\right) d\right]\right\}
$$

in which the parameter $d$ is related to the distance between the tails of the function, $i$ a value related to the asymmetry of the bell profile, $P_{\mathrm{m}}$ the maximum protected molecules of the substrate used in the reaction ( $\beta \mathrm{C}$ and $\mathrm{Cr}$ in this case) and $t_{\mathrm{m}}$ the time at which $P_{\mathrm{m}}$ takes place.

This model explicitly provides the characterizing parameters $\left(P_{\mathrm{m}}\right.$ and $\left.t_{\mathrm{m}}\right)$ of the RD response, and therefore their statistical significance can be tested through the determination of its confidence intervals. Figure 2 (A1 and B1 plots) shows the application of this model to predict the effect of $\mathrm{BHT}$ and $\mathrm{Fe}^{+2}$ in the $\beta \mathrm{C}$ reaction. All the parametric values are presented in appendix section (Table A1 and Table A2), showing lower confidence intervals $(\alpha=0.05)$ and higher correlation coefficients in all cases $\left(r^{2}>0.99\right)$, thus demonstrating the reliability of this approach. The two characterizing parameters $\left(P_{\mathrm{m}}\right.$ and $\left.t_{\mathrm{m}}\right)$ will vary in the presence of any antioxidant and, given their well-defined factual meanings regarding the oxidation kinetics, their combine variations have a relevant characterizing value.

On the one hand, plotting the $P_{\mathrm{m}}$ parameter against $\mathrm{OM}$ concentration show an asymptotic trend (Figure 2, A2 and B2 plots), suggesting that some radical-generating property of the system can be saturated (Gieseg \& Esterbauer, 1994). This type of dose-response patterns, in general, can be adjusted to the following asymptotic function:

$$
P_{m}(O M)=K[1-\exp (-r[O M])]
$$

where $[\mathrm{OM}]$ is the concentration of the $\mathrm{OM}$ agent under study in $\mu \mathrm{M}, P_{m}(\mathrm{OM})$ is the response behavior of the parameters $P_{m}$ as a function of [OM], $K$ is the asymptotic value of the parameter 
obtained ( $\mu \mathrm{M}$ of the protected substrate) and $r$ is the specific dose-rate $\left(\mu \mathrm{M}^{-1}\right.$ of OM). If the OM agent is an antioxidant the response will be positive and negative for pro-oxidants.

On the other hand, the $t_{m}$ parameter shows a linear dose-response trend (Figure 2, A3 and B3 plots) with an intercept that can be easily adjusted to:

$t_{m}(O M)=t_{0}+b[O M] Q=K \times r$

where $b$ is the slope $(\mathrm{min} / \mu \mathrm{M}$ of $\mathrm{OM})$ of the dose-response trend and $t_{0}$ is the extension time (min) at which the lipid change oxidation reaction behaves in the absence of any OM, in other words the extension time produced by $1 \mu \mathrm{M}$ of $\beta \mathrm{C}$ (the competitor antioxidant). If the $\mathrm{OM}$ agent is an antioxidant the linear response will be positive increasing and decreasing for pro-oxidants.

The resulting kinetic parameters, obtained after the fitting procedure to equation [2], are adjusted to their respective equations [3] and [4] as a function of [OM], obtaining in all cases highly consistent results with satisfactory confidence intervals $(\alpha=0.05)$.

\subsubsection{Simple analytical criteria to compare the time-dose response of compounds}

In addition, after obtaining the parametric estimates of equations [3] and [4], it is possible to summarize the time-dose response in two complementary single values (the $Q$ and $S$ values).

The $Q$ value, which corresponds to the amount of molecules protected per unit of $\mathrm{OM}(\mu \mathrm{M}$ of the protected substrate/ $\mu \mathrm{M}$ of $\mathrm{OM})$ at the moment of maximum predicted capacity, is calculated by multiplying both parameters $(K$ and $r$ ) estimated by equation [3] as follows:

$Q=K \times r$

In the case of $S$ value, its determination is performed following the next procedure: First, to compute the OM concentration needed at any percentage of the response by equation [3], the $P_{m}$ (OM) is considered to be $P_{m}=K \times n / 100$, in which $n$ can be any value between $0-100 \%$, consequently the corresponding $[\mathrm{OM}]_{n}$ can be computed to obtain any $n$ percentage of the maximum $\mu \mathrm{M}$ of the substrate protected $P_{m}(\mathrm{OM})$ by the following expression:

$[O M]_{n}=-\frac{\operatorname{Ln}(1-(n / 100))}{r}$

Then, by inserting this $[\mathrm{OM}]_{n}$ to reach $n$ percentage of the protected substrate into equation [4], the protection time until the substrate reaches this $n$ percentage can be obtained as:

$$
S_{n}=t_{0}+b[O M]_{n}
$$

in which $t_{0}$ and $b$ are the parameter estimates previously computed by equation [4]. Even if the typical approach is to consider the half-life response or in this case $n=50 \%$, it would be appropriate to compute the $S$ value for the concentration needed to reach the asymptotic value of equation [3] ( $K$ or $100 \%$ of the response), complementing accordingly the information provided by the $Q$ value. However, when computing the $S$ value for $n=K$, the $[\mathrm{OM}]_{n=K}$ will be excessively high, in occasionally outside of the kinetic range capabilities or extending the assay 
inappropriately. Therefore the $95 \%$ value was considered the more suitable response (Figure 2, $\mathrm{A} 2$ and $\mathrm{B} 2$ plots).

These values can be used to compare the activities of different OM agents. For example, the $Q$ value of BHT showed that the maximum capability of one molecule is to protect 0.10 molecules of $\beta \mathrm{C}\left(0.10 \mu \mathrm{M} \beta \mathrm{C}\right.$ protected $/ \mu \mathrm{M}$ of BHT), on the other hand, the $S_{n=95 \%}$ for BHT showed that at the $95 \%$ of its maximum capabilities the protection time was 89.23 min (knowing that $\left.[\mathrm{BHT}]_{n=95 \%}=20.51 \mu \mathrm{M}\right)$. The information provided by the combination of both values represents a robust tool to compare the activities of different antioxidant agents based on the parametric estimations time-dose response. With both values, an intuitive solution to compare OM activities of compounds by a mathematical analysis is obtained, offering researchers an alternative solution based on parametric non-linear values to assess OM action and compare their capacity rigorously. Furthermore, the application may facilitate the ranking process and the selection of appropriate concentrations of natural products to replace commercial antioxidants.

\subsection{Verification of the quantification procedure when applied to assess and compare several $O M$ agents in two different competitive assays}

\subsubsection{Antioxidants}

Figure 3A and Table A1 (appendix) show the graphic representations of the results and the parametric estimates of the time-dose fittings of equation [2] to the results of the proposed approach for the $\beta \mathrm{C}$ bleaching reaction applied to five common commercial antioxidants. Figure 3B and Table A3 (appendix) show the corresponding results of the proposed approach for the $\mathrm{Cr}$ bleaching reaction. Table 1, the parametric estimates of equations [3] and [4] obtained after fitting the parametric results ( $P_{m}$ and $t_{m}$ parameters) from equation [2] are shown for both assessed reactions. It is particularly noteworthy to point out that for both reactions, only for the case of ETX in the $\beta \mathrm{C}$ system, the maximum substrate protected $\left(P_{m}\right)$ reaches an asymptotic value $(K)$ equal to the total amount of $\beta C$ present in the final solution of the reaction.

Furthermore, the computed criteria values $Q$ and $S$ to compare the antioxidant capacity are presented in Table 1 and Figure 3 (A2 and B2 plots):

In the $\beta C$ reaction, the value $Q$ for the compound ETX was found to protect 291.2 molecules of the substrate $\beta C$ per molecule of antioxidant, which is by far the highest value reached, followed by TOC with 20.62. With regards to the time at which the maximum protection took place, the value $S$ again show that ETX protected the oxidation of $\beta$ C (139.9 min) for longer periods than the others, such as TOC with $121.9 \mathrm{~min}$.

- In the Cr reaction the differences between the antioxidants assessed were less than in the $\beta C$ reaction. Nevertheless, the antioxidant ETX showed the best criteria values than compared to any of the other compounds tested.

The combined criteria values, provide complementary information to compare the capacity of different compounds. Beyond quantitative differences, the following ranking of their capacity can be established:

For the $\beta C$ reaction: ETX $>>$ TOC $>>$ BHA $>$ BHT $>$ PG.

For the Cr reaction: ETX $>\mathrm{Mn}^{+2}>\mathrm{Tr}>\mathrm{AA}>\mathrm{TBHQ}$.

\subsubsection{Pro-oxidants}

Numerous agents such as transition metals can directly or indirectly catalyze the oxidative mechanisms in both lipophilic and hydrophilic environments. As a possible example of pro- 
oxidant activity, some transition metals are selected to test the method proposed. The effects on different systems is not less relevant than those of commercial antioxidants, since they can be present, either as constituents or contaminants, in many extract materials and as traces in buffer salts, thus distorting the results. Figure 3C and Table A2 (appendix) show the graphic representations of the results and the parametric estimates of the time-dose fittings of equation [2] to the results of the proposed approach for the $\beta \mathrm{C}$ and $\mathrm{Cr}$ bleaching reactions applied to four common commercial antioxidants. In Table 1, the parametric estimates of equations [3] and [4] obtained after fitting the parametric results $\left(P_{m}\right.$ and $t_{m}$ parameters) of equation [2] are shown for both assessed reactions.

The amount of reduced hemoglobin used, which refers to hemoglobin (considering an average of of $64,500 \mathrm{kDa}$ per molecule) which contains iron in the $\mathrm{Fe}^{+2}$ oxidation state, had the approximately the same quantity of $\mathrm{Fe}^{+2}$ as the amount introduced directly as iron (II) sulfide. In fact, the parametric response (Table 1) as well as the graphical representation of the results (Figure 3C) are approximately equivalent, demonstrating the reliability of the tools here developed.

\subsection{Extension of the model application to the combine effect of an antioxidant and a pro- oxidant agent}

One of the additional features of the developed approach is that can be easily extended to a more complex situations, that occasionally are experimentally found. For instance, when testing the OM activity of natural compounds is likely to expect responses that can be a combination of some antioxidants and pro-oxidants. Such responses cannot be directly analyzed by the usual approaches, and therefore to identify the joint activity of each OM compound certain further steps need to be executed.

As example, the combinatory analysis of the antioxidant BHT and the pro-oxidant $\mathrm{Fe}^{+2}$ in the $\beta \mathrm{C}$ assay will be presented. A $6 \times 10$ arrays of an increasing concentrations of a mixture of an antioxidant and a pro-oxidant, in which $25 \mu \mathrm{L}$ of each OM solution are added to each well containing $250 \mu \mathrm{L}$ of the preheated reagent and the other conditions were kept. A total of 30 independent kinetic measures per each of the 60 concentration combinations were obtained and are displayed in Figure A1 (appendix section). It can be seen that as the concentration of prooxidant increases the oxidation of $\beta C$ increases and the effect of the antioxidant becomes less effective. The temporal space of action $\left(t_{m}\right)$ of the pro-oxidant compound is earlier than for the antioxidant, causing biphasic curves caused by its interaction. To analyze such a response additive equations must be used increasing the number of parameters, which makes more difficult the interpretation of the results.

When the effects are displayed in terms of RD (using equation [1]) in Figure 4A (BHT time-dose response for three pro-oxidant concentrations), depending on the range of concentrations used for each compound, only antioxidant activity is seen, which are the curves in the positive axes, only pro-oxidant activity (curves in the negative axis) or both actions when the curves goes from one axis (negative or positive) to the other. The application of the RD standardization allows to visually detect the opposite actions of both agents and provide a quick overall output of the final interaction. However, its analytical determination also requires the sum of two independent equations (one for each OM) as the one described in [2]. As well as if we applied other common resources to the raw data (Figure A1, appendix) a high number of parameters are needed, and depending on the profile of the curve some of them will be non-statistically significant due to the lack of effect. Therefore, the outputs obtained by modeling those types of profiles must be rejected. 
However, since the RD is based on the subtraction of the control, it can be consider that the effect of one of the $\mathrm{OM}$ as a function as other as a type of control subtracting its effect, thus reducing the number of variables. In Figure 4B the effect of the each concentration of $P$ is subtracted to the antioxidant time-dose response, allowing to analyze the entire set of responses by equation [2] producing statistically significant parametric results (Table A4). The subtraction of the effect of the $P$ only simplifies the operational procedure, and still possible to quantify the interactive effects by determining the parametric values $P_{m}$ and $t_{m}$. Since both values are affected by the interaction of two OM, the univariate equations [3] and [4] ( $P_{m}$ and $t_{m}$, respectively) can be expanded to perform a much consistent approach taking into account both effects simultaneously by the following bivariate analysis:

$$
\begin{aligned}
& P_{m}(A, P)=K\left\{1-\exp \left[-r_{A}[A] \exp \left(-r_{P}[P]\right)\right]\right\} \\
& t_{m}(A, P)=t_{0}+b_{A}[A]+b_{P}[P]
\end{aligned}
$$

Figure $4 \mathrm{C}$ shows the univariate results (points) and the fitting to the bivariate equations [8] and [9] (surface). The parametric results of the bivariate analysis of $P_{m}$ are $K=0.717 \mu \mathrm{M}$ of the substrate protected, $r_{A}=0.667 \mu \mathrm{M}^{-1}$ of BHT and $r_{P}=0.213 \mu \mathrm{M}^{-1}$ of $\mathrm{Fe}^{+2}$ with a $\mathrm{r}^{2}=0.9927$. On the other hand, the parametric results of $t_{m}$ are $t_{0}=37.10 \mathrm{~min}, b_{A}=8.001 \mathrm{~min} / \mu \mathrm{M}$ of BHT and $b_{P}=3.912 \mathrm{~min} / \mu \mathrm{M}$ of $\mathrm{Fe}^{+2}$ with $\mathrm{a} \mathrm{r}^{2}=0.9862$.

\subsection{Verification of the quantification procedure with experimental data from other competitive methods}

The bibliographical abundance about antioxidant activity in a competitive reaction, in raw and purified extracts, makes it practically superfluous to extend the experimental work specifically devoted to validate the model proposed here. In this respect, its descriptive accuracy was verified using results from other authors (taken from the published figures by means of GetData Graph Digitizer 2.24), selected in such a way that they implied different methods, substrates and time domains.

\subsubsection{Oxidative hemolysis inhibition assay (OxHLIA)}

The method is based in the oxidation of erythrocyte membranes by AAPH-derived peroxyl radicals that induces oxidation of lipids and proteins and eventually causes hemolysis, and this hemolysis can be inhibited by antioxidants. OxHLIA is a good experimental model for free radical-induced biomembrane damage and its inhibition by antioxidants. Figure 5 (A1 plot) shows the typical time-dose response of hemolysis curves using the antioxidant $\operatorname{Tr}$ at various concentrations 0-(25)-125 mM. The results were obtained from the study of Takebayashi et al. (2010) who recently made a detail revision of the method. Figure 5 (A2 plot) shows the fittings (lines) of the equation [2] to the data in terms of RD (points). Figure 5 (A3 plot) and Table 1 shows the parametric results equations [3] and [4] ( $P_{m}$ and $t_{m}$, respectively). Furthermore the computed criteria values $Q$ and $S$ to compare the antioxidant capacity are presented in Table 1 .

\subsubsection{Oxygen radical absorbance capacity assay (ORAC)}

Currently, this method has been automated and transferred to a microplate format producing a large amount of dose-time-data effortless. The assay depends on the free radical damage to the fluorescent compound of fluorescein, which acts as the indicator of the reaction, changing its 
fluorescent intensity. It is assumed that the degree of change is indicative of the amount of radical damage. The addition of antioxidants results in a competitive inhibition in the free radical damage to the fluorescent compound. The data was obtained from the work of Ou et al. (2001) who developed and validated the assay. Figure 5 (B1 plot) shows the typical time-dose response fluorescein decay curves in the presence $0-(0.05)-0.2 \mathrm{mg} / \mathrm{L}$ of grape seed extract. Figure 5 (B2 plot) shows the fittings (lines) of the equation [2] to the data in terms of RD (points). Figure 5B3) and Table 1 shows the parametric results equations [3] and [4] ( $P_{m}$ and $t_{m}$, respectively). Furthermore the obtained values $Q$ and $S$ to compare the antioxidant capacity are also summarized in Table 1.

\section{DISCUSSION}

Perhaps, the biggest problem is related to the lack of a validated assay that can reliably measure the antioxidant and pro-oxidant capacity of samples, thus making it essential to test the capacity with different methods. As a result, authors tend to simplify the calculation method in order to amplify the number of testing procedures. However, the method used to measure and compute the antioxidant capacity has a major impact on the results, because in both in vivo and in vitro, the oxidation reactions are complex. The abbreviated approach to study the dose-response at one single-time expecting to find linear forms (as described by the non-kinetic approaches) frequently leads to unreliable results and misinterpretations, making it extremely difficult to compare the results from different assays. The preference of apparently simple assays, routinely applicable with minimal calculation requirements, is not very justifiable today, given the availability of computational applications and automatic equipment (such as microplate readers), whose combination provides adequate tools to work with data sets that allow accurate evaluations by the available non-linear modeling (Labuza \& Dugan, 1971; Murado \& Vázquez, 2010; Terpinc \& Abramovič, 2010; Wardhani et al., 2013; Özilgen \& Özilgen, 1990). Despite the advisability of using mechanistic or empiric kinetic models as indicated by different authors, researchers continue to use simple calculation alternative methods more often than necessary.

The detailed mechanistic description of lipid oxidation is complex and varies from one to the other systems, which has led to the search for empirical general models, able to describe the most common profiles. In this sense, among the available non-linear models to describe the time part of an oxidative reaction individually for increasing concentrations of the OM agent, may also be subjected to analysis. For example, the power function developed by Terpinc \& Abramovič, (2010) is appropriate only to adjust fractional-order kinetic profiles, but fails in the description of first-order processes or sigmoidal profiles. Other empirical approaches such as the Logistic and Weibull equations, that have been transferred from other fields to describe the oxidation action (Murado \& Vázquez, 2010; Özilgen \& Özilgen, 1990), are more appropriate for modeling processes as the lipid oxidation. Those equation are able to produce key parameters to summarize the responses, such as the asymptote, maximum velocity or the lag-phase, they can characterize the response and help to quantify the effect of OM agents. In general, the three parameter sigmoidal group of functions (such as the Logistic, Weibull, Hill, Gompertz or Richards-Chapman) is the best solution to fit individually the kinetic profiles corresponding to a series of increasing levels of OM agents. Alike in many other complex systems, some authors (Murado \& Vázquez, 2010; Prieto et al., 2013a; 2013b) have suggested directly or indirectly further analysis, in which the oxidative responses are described as a function of both the dose and the exposure time, in a bivariate form.

Our proposal represents an alternative for the dose-time-response behavior, based on two kinetic parameters of equation [2], which jointly defines the capacities of the OM to extend or shorten the maximum protection as a function of the concentration. In fact, it is able to describe 
accurately different rate-dose tendencies. It allows quantification of the variations of the kinetic profiles which characterize the different types of antioxidants in a useful way that can provide even indications concerning modes of action. Independently of the mechanistic interpretation that can be inferred by analyzing the specific behavior of both characterizing parameters, in competitive assays, the time dependent bell protection function produces consistent and meaningful criteria for comparative characterization and quantification of any antioxidant, in a dose-time frame which minimizes the effects of the error produced by the experimental conditions.

Additionally, by standardizing the response using the equation [1] the results obtained do not depend on the experimental conditions, particularly on the initial concentration of the reactive species, which is in practice, one of the common problems when analyzing the efficacy of an antioxidant in competitive methods. In a competition assay, it has to be realized that during the assay the concentration of the antioxidant as well as that of the indicator of the reaction can be reduced to a considerable extent. The consumption of both during the experiment, as an inevitable consequence of the competition that has to take place, is a potential cause of inaccurate results (Balk et al., 2009).

In this work, we have clearly demonstrated the capabilities of the model to discern the effects of several commercial agents providing useful information in the study of complex natural extracts containing components with variable degrees of OM capacity. For all the assayed agents, statistically significant descriptions, with very accurate predictions, were provided by model [2]. In the presence of antioxidants or pro-oxidants, the molecules of the substrate protected and the time at what takes place increases according to equations [3] and [4]. This variation is general enough to explain the alteration of the kinetic profile due to the presence of an OM compound.

\section{CONCLUSIONS}

The complexity of the topic of antioxidants and pro-oxidants plus the confusion introduced by improper use of questionable methods leads to the disarray of the antioxidant research community and industry. In this paper, a quantification method was developed for competitive assays and tested by investigating the capacity of several antioxidants in different competitive systems. The analysis of the antioxidant capacity of commercial antioxidants reveals the lack of meaning of single-time criteria and the possibilities of the proposal presented. The model parameters obtained were used to compare the capacity, identifying complex trends and analyzing the dose-equivalent system response, providing more complete information about antioxidant behavior and a more efficient way to determine the total antioxidant capacities that those techniques at a fixed point.

\section{AKNOWLEDGEMENTS}

The authors wish to thank CSIC (Intramural project: 200930I183) and Ministerio de Ciencia e Innovación (project CTM2010-18411, co-financed with FEDER funds by European Union) for financial support. Miguel Ángel Prieto Lage was awarded one grant from the JAE predoctoral program co-financed by the CSIC and European Social Fund (ESF). 


\section{FIGURES}

\section{A: ANTIOXIDANT ACTION}

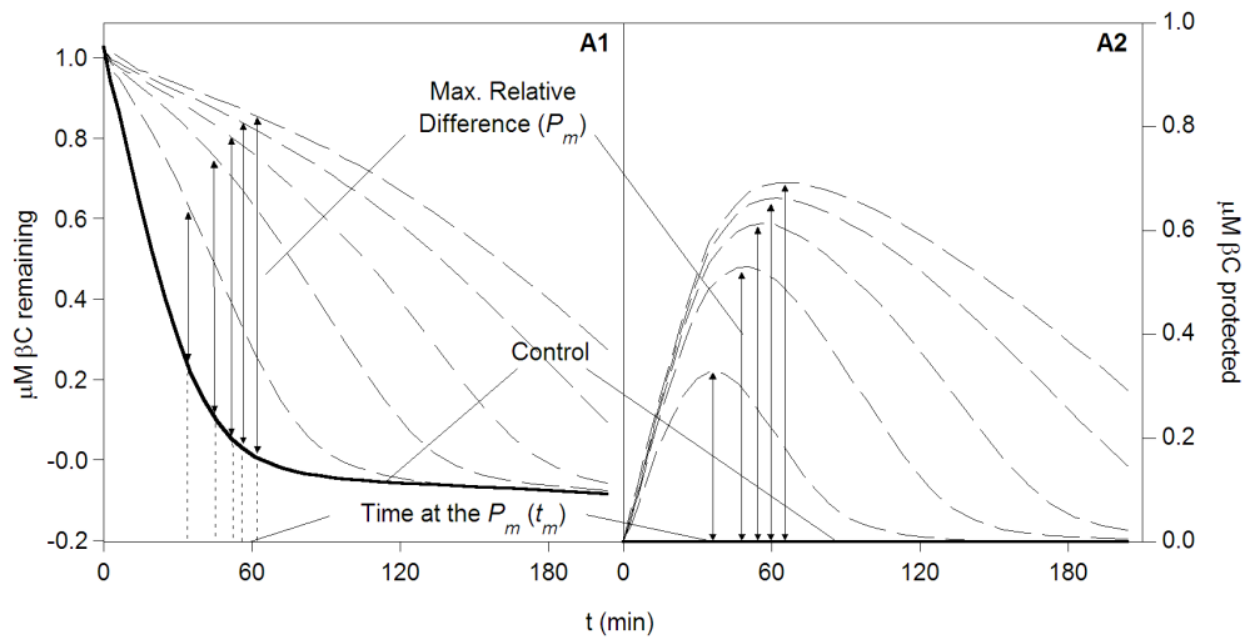

B: PRO-OXIDANT ACTION

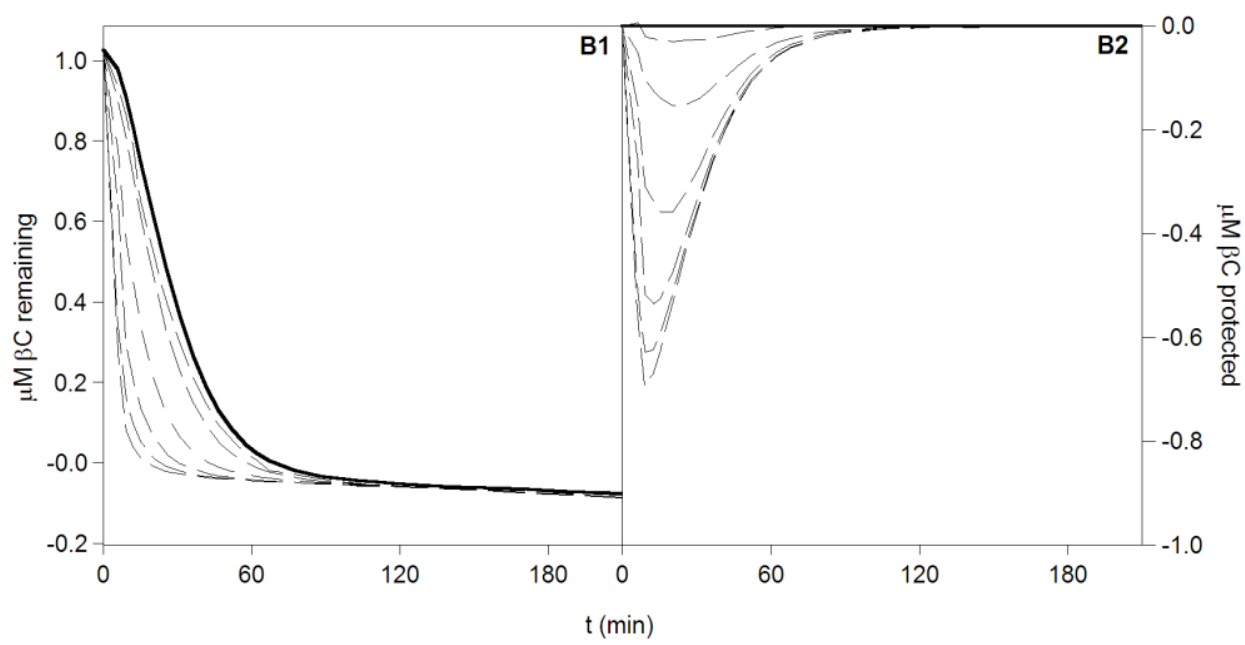

Figure 1: Illustrative representation of the characteristic profiles obtained for antioxidant (A) and pro-oxidant (B) responses using equation [1] to standardize in the $\beta$-carotene $(\beta C)$ bleaching reaction as examples. $\mathrm{A} 1$ and $\mathrm{B} 1$ show the raw responses of the $\beta \mathrm{C}$ reaction as function of time and $\mathrm{A} 2$ and $\mathrm{B} 2$ the asymmetric bell profile of the kinetic relative difference response. 
A: ANTIOXIDANT ACTION

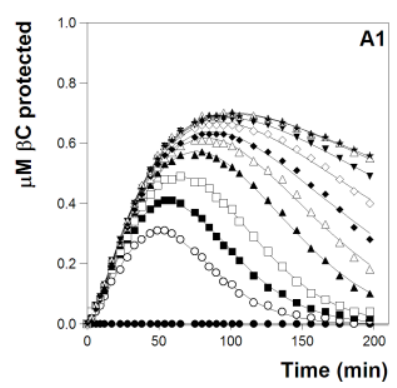

B: PRO-OXIDANT ACTION

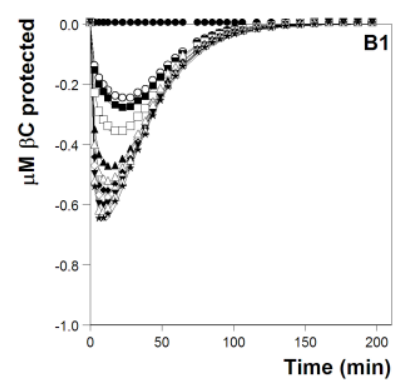

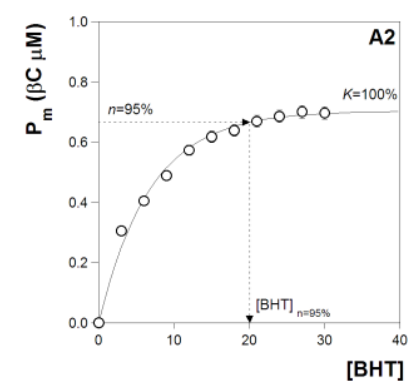
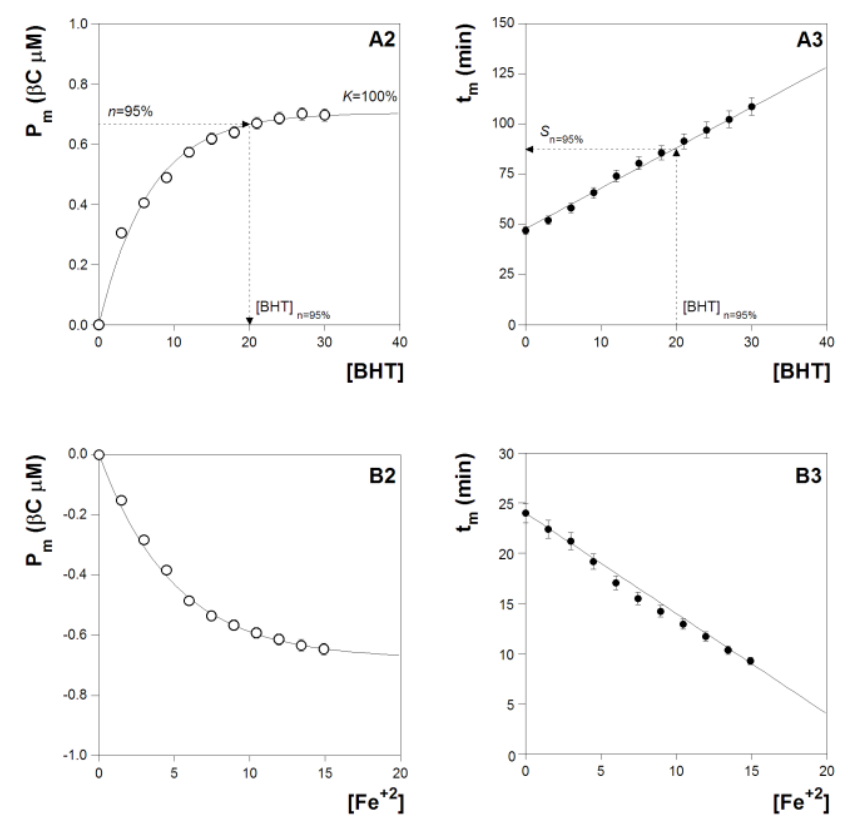

Figure 2: The kinetic parameters that could characterize the response the maximum protected molecules of $\beta \mathrm{C}\left(P_{\mathrm{m}}\right)$ and the time at which it takes place $\left(t_{\mathrm{m}}\right)$ are displayed. A1 and B1 show the fittings to the asymmetric bell profile of the kinetic relative difference dose-response of the examples presented in Figure 1 to the model [2]. A2 and B2 show the maximum protected molecules of $\beta \mathrm{C}\left(P_{\mathrm{m}}\right)$ fitted to the equation [3]. A3 and B3 display the time at which it takes place $\left(t_{\mathrm{m}}\right)$ the $P_{\mathrm{m}}$ fitted to the equation [4]. For all cases, the points are the findings and the lines are the fitted results to the corresponding model. All numerical results in Table 1, Table A1 and Table A2. 


\section{A: $\beta C$ assay}

A1: TIME-DOSE ANALYSIS
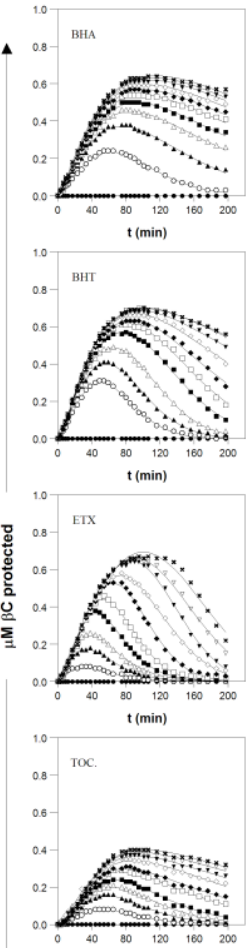

$t(\min )$
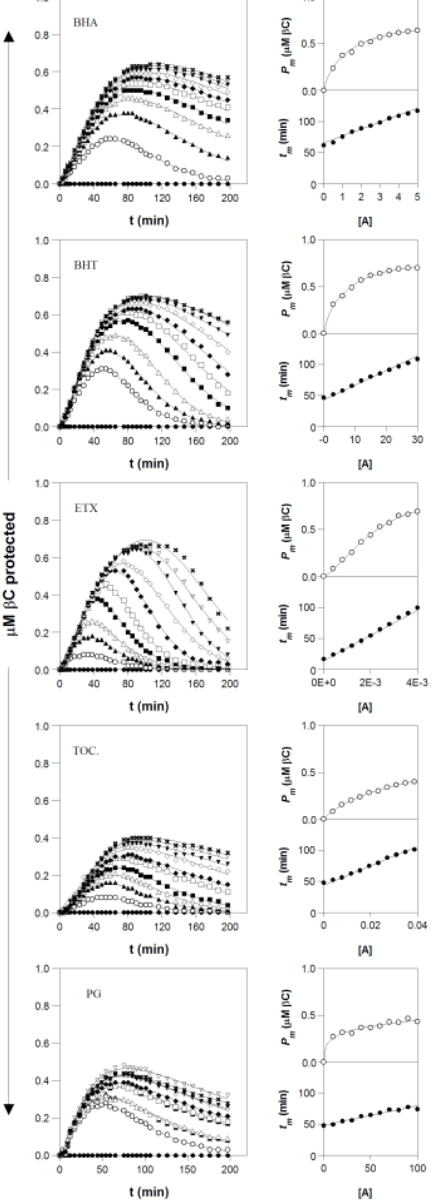

[A]
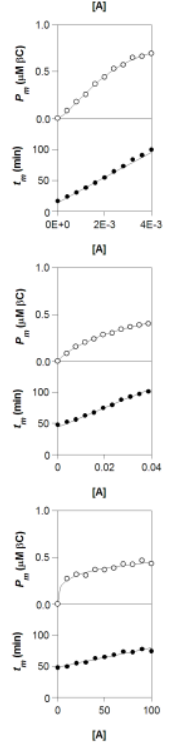

A2: CRITERIA VALUES FOR COMPARISON

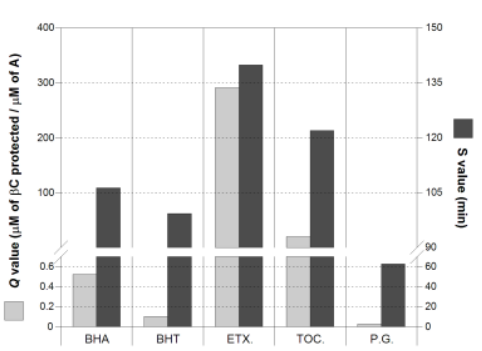

Antioxidants tested

\section{B: Cr assay}

B1: TIME-DOSE ANALYSIS
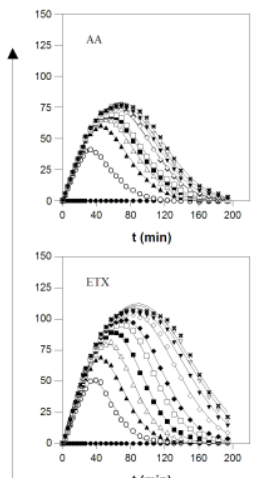

(min)
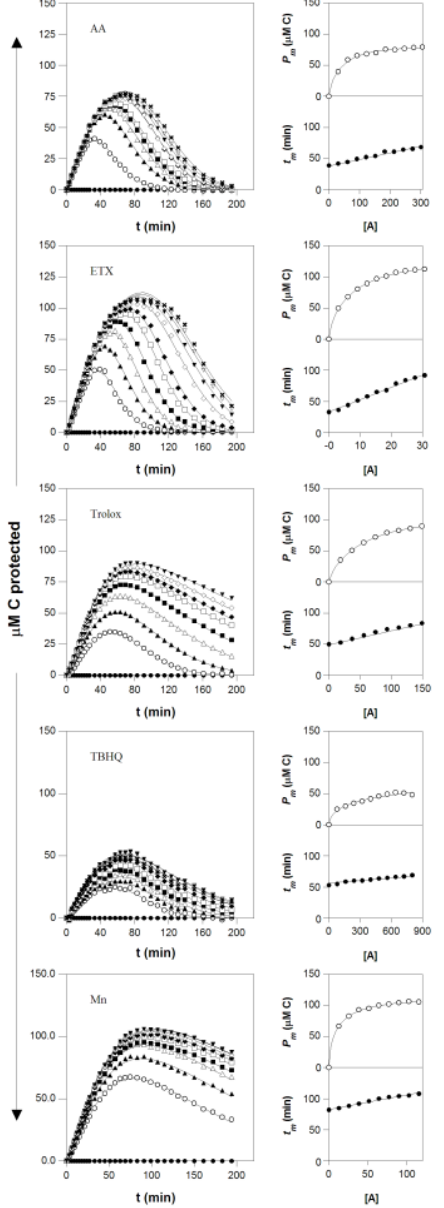

B2: CRITERIA VALUES FOR COMPARISON

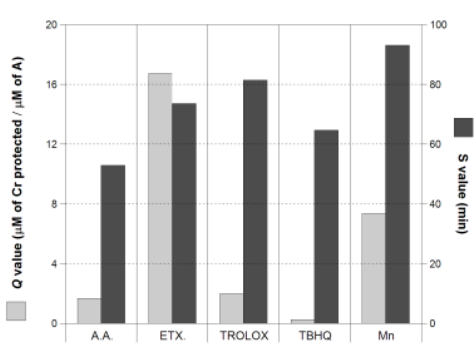

Antioxidants tested

\section{$\mathrm{C}: \boldsymbol{\beta C}$ and $\mathrm{Cr}$ assays}

C1: TIME-DOSE ANALYSIS OF $\beta C$ ASSAY
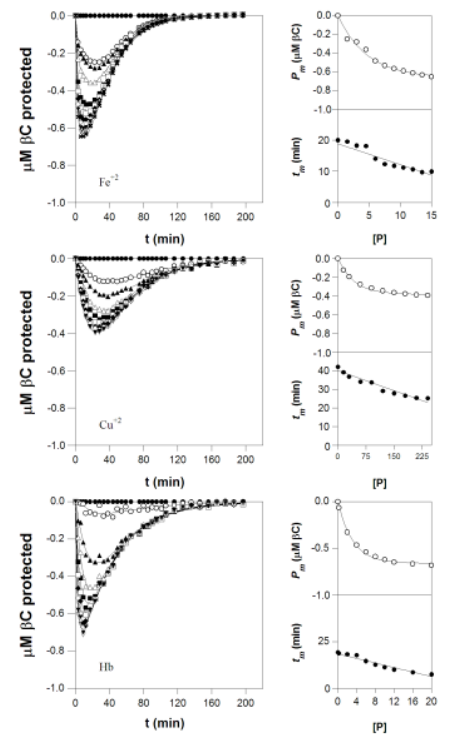

C2: TIME-DOSE ANALYSIS OF CROCIN ASSAY
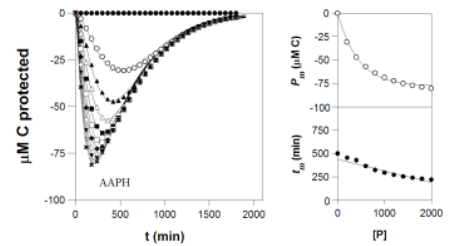

C3: CRITERIA VALUES FOR COMPARISON

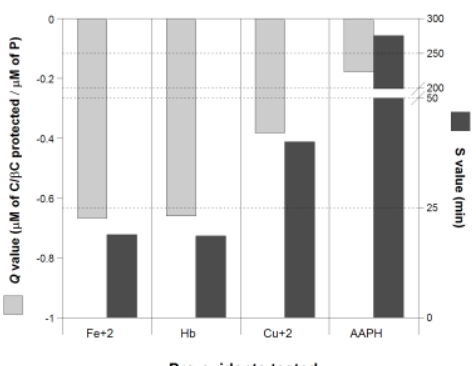

Pro-oxidants tested

Figure 3: Experimental results for the $\beta$-carotene and crocin bleaching reaction. Each figure of the OM analysis is divided as follows: on the left side, the time protection profiles drop orderly with the increase of the agent concentrations and are fitted to equation [2] and on the right side, the $P_{m}$ and $t_{m}$ parameters pattern are shown and fitted to the equations [3] and [4] respectively. Figures in the sub-sections $\mathrm{A} 1, \mathrm{~B} 1, \mathrm{C} 1$ and $\mathrm{C} 2$ show the effects of several antioxidants and prooxidants obtained in the $\beta C$ and $\mathrm{Cr}$ bleaching assays. Sub-sections A2, B2 and C3 show the results of the analytical criteria values ( $Q$ and $S$ ) used to compare the capacity of OM.. Experimental results are points and fittings to the corresponding models are lines. All numerical results are in Table 1, Table A1, Table A3 and Table A2. 
A: Standardize with the global control response
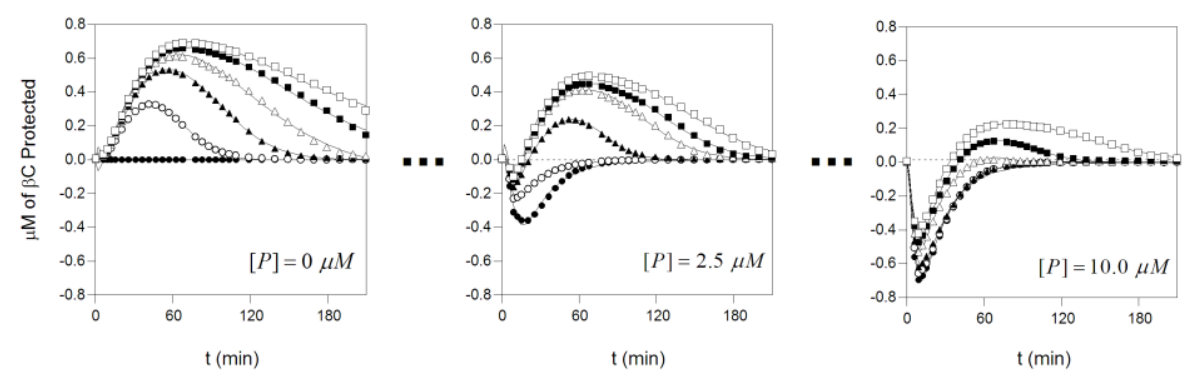

B: Standardize using the control response for each specific [P]

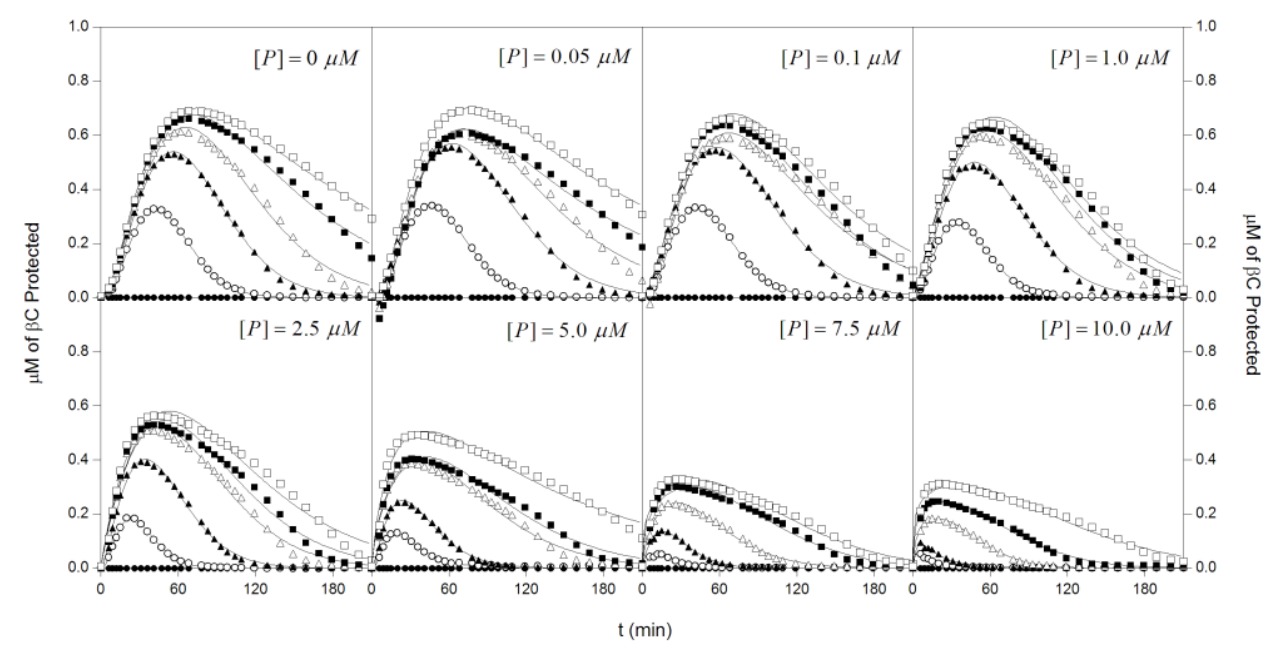

C: Bivariate analysis of the $P_{m}$ and $t_{m}$ parameters
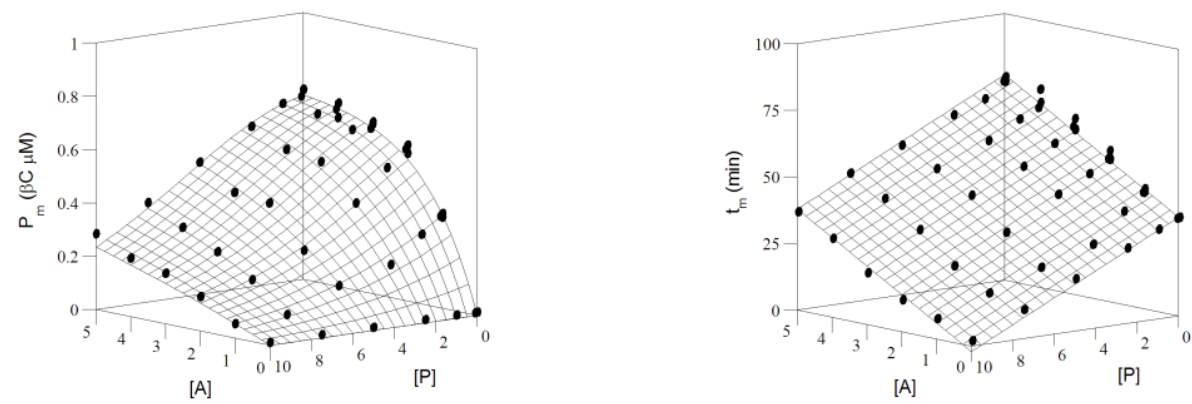

Figure 4: Analysis of the combine action of an antioxidant and a pro-oxidant. All numerical results are in Table A4. The parametric results of the bivariate analysis are described in the text. 

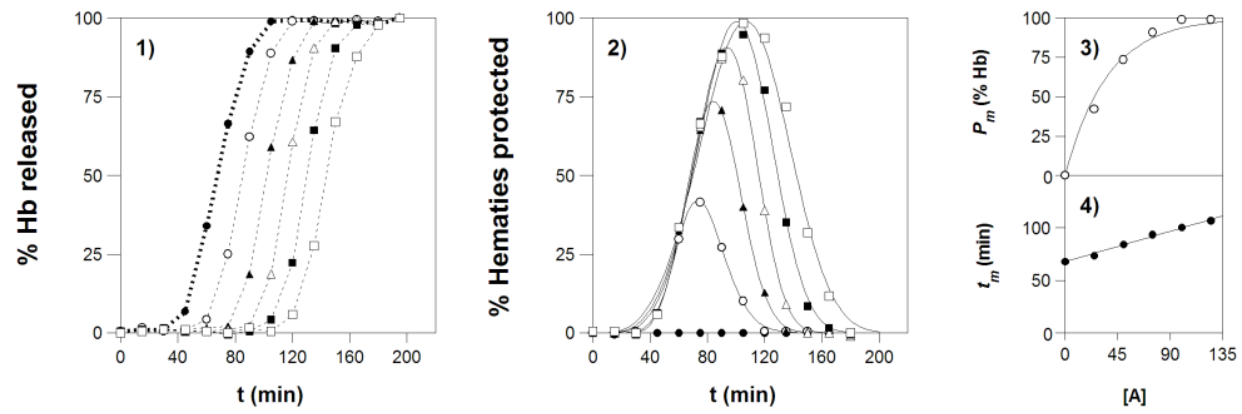

\section{B: ORAC ASSAY}
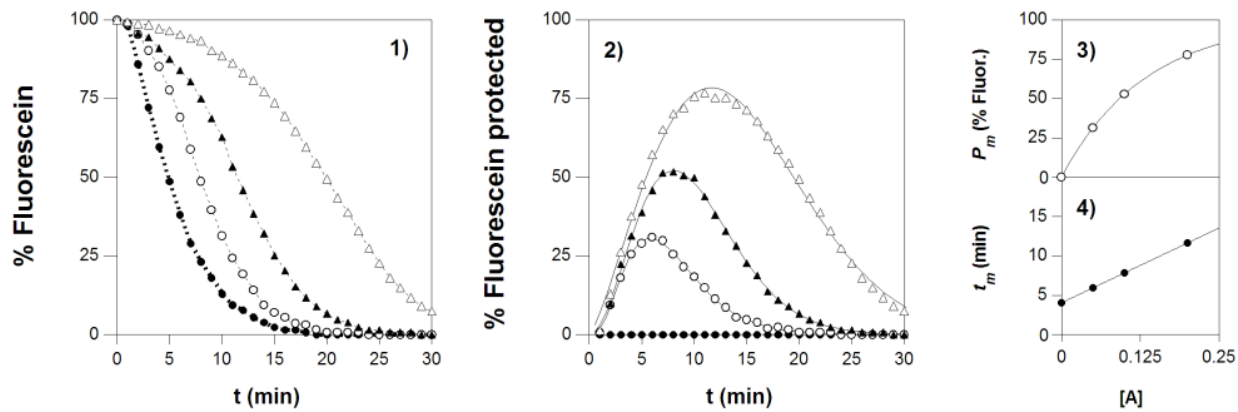

Figure 5: Analysis of other potential methodological applications. A: OxHLIA assay data, obtained from Takebayashi et al. (2010) that shows the typical time-dose response hemolysis curves of Trolox $(0 \bullet, 25 \bigcirc, 50 \boldsymbol{\Delta}, 75 \triangle, 100 \square$ and $125 \square \mathrm{mM}$ using sheep erythrocytes suspended at a concentration of $0.7 \%(\mathrm{v} / \mathrm{v})$ in PBS incubated at $37^{\circ} \mathrm{C}$ with $40 \mathrm{mM}$ of AAPH. B: ORAC assay data, obtained from $\mathrm{Ou}$ et al. (2001) showing the fluorescein decay curve induced by AAPH in the presence of $0 \bullet, 0.05 \bigcirc, 0.1 \Delta$ and $0.2 \triangle \mathrm{mg} / \mathrm{L}$ of grape seed extract. Analytical criteria values $(Q$ and $S)$ used to compare the capacity among several antioxidants are presented in Table 1. All numerical results are in Table A5 (appendix). 


\section{TABLES}

Table 1: Parametric estimates of equations [3] and [4] obtained after fitting the parametric results ( $P_{m}$ and $t_{m}$ parameters) from equation [2] for the crocin and $\beta$-Carotene bleaching kinetics as affected by the specified agents respectively. Also the analytical criteria values $(Q$ and $S)$ used to compare the capacity among several antioxidants are shown. The confidence intervals $(\alpha=0.05)$ are in percentages.

\begin{tabular}{|c|c|c|c|c|c|c|c|c|}
\hline \multirow{2}{*}[\mathbf{OM}]{} & \multicolumn{3}{|c|}{ Parameters of $P_{m}(\mathrm{OM})$} & \multicolumn{3}{|c|}{ Parameters of $t_{m}(\mathrm{OM})$} & \multicolumn{2}{|c|}{ Criteria values } \\
\hline & $K$ & $r$ & $\mathbf{r}^{2}$ & $t_{0}$ & $b$ & $\mathbf{r}^{2}$ & $Q$ & $S$ \\
\hline \multicolumn{9}{|c|}{ B-CAROTENE ASSAY } \\
\hline BHA & $0.635 \pm 4.0$ & $0.827 \pm 6.1$ & 0.9990 & $69.03 \pm 3.0$ & $10.28 \pm 6.1$ & 0.9992 & $0.525 \pm 8.4$ & $106.2 \pm 2.2$ \\
\hline BHT & $0.700 \pm 3.2$ & $0.146 \pm 1.2$ & 0.9996 & $47.97 \pm 1.1$ & $2.011 \pm 5.6$ & 0.9994 & $0.102 \pm 3.8$ & $89.23 \pm 0.7$ \\
\hline ETX. & $1.000 \pm 3.2$ & $291.2 \pm 2.3$ & 0.9988 & $47.28 \pm 1.3$ & $9002 \pm 3.4$ & 0.9991 & $291.2 \pm 7.4$ & $139.8 \pm 3.2$ \\
\hline TOC. & $0.481 \pm 2.7$ & $42.87 \pm 3.1$ & 0.9981 & $58.15 \pm 3.2$ & $912.4 \pm 4.4$ & 0.9990 & $20.61 \pm 8.4$ & $121.9 \pm 4.8$ \\
\hline P.G. & $0.421+2.2$ & $0.064 \pm 4.1$ & 0.9984 & $48.48 \pm 5.3$ & $0.309 \pm 3.5$ & 0.9981 & $0.027 \pm 9.0$ & $62.94 \pm 4.1$ \\
\hline $\mathrm{Fe}^{+2}$ & $-0.668 \pm 2.3$ & $0.212 \pm 1.5$ & 0.9880 & $18.87 \pm 2.8$ & $-0.671 \pm 3.1$ & 0.9991 & $-0.668 \pm 5.6$ & $18.87 \pm 4.4$ \\
\hline $\mathrm{Hb}$ & $-0.659 \pm 1.3$ & $0.313 \pm 2.2$ & 0.9921 & $18.55 \pm 4.5$ & $-0.610 \pm 4.4$ & 0.9955 & $-0.660 \pm 3.1$ & $18.55 \pm 6.6$ \\
\hline $\mathrm{Cu}^{+2}$ & $-0.383 \pm 7.8$ & $0.022 \pm 3.4$ & 0.9902 & $41.10 \pm 2.6$ & $-0.071 \pm 3.6$ & 0.9976 & $-0.383 \pm 7.8$ & $40.00 \pm 5.1$ \\
\hline \multicolumn{9}{|c|}{ CROCIN ASSAY } \\
\hline A.A. & $76.62 \pm 5.1$ & $0.022 \pm 3.3$ & 0.9970 & $38.06 \pm 3.1$ & $0.109 \pm 4.4$ & 0.9989 & $1.685 \pm 6.8$ & $52.90 \pm 2.2$ \\
\hline ETX. & $100.0 \pm 3.4$ & $0.150 \pm 2.2$ & 0.9997 & $33.59 \pm 2.3$ & $2.001 \pm 1.3$ & 0.9986 & $16.72 \pm 7.5$ & $73.55 \pm 1.1$ \\
\hline TROLOX & $91.12 \pm 1.7$ & $0.022 \pm 1.3$ & 0.9977 & $51.69 \pm 1.5$ & $0.218 \pm 0.9$ & 0.9989 & $2.004 \pm 2.2$ & $81.37 \pm 1.0$ \\
\hline TBHQ & $50.03 \pm 2.8$ & $0.005 \pm 1.7$ & 0.9976 & $56.32 \pm 7.8$ & $0.014 \pm 1.5$ & 0.9980 & $0.250 \pm 4.8$ & $64.71 \pm 6.8$ \\
\hline $\mathrm{Mn}^{+2}$ & $100.0 \pm 4.5$ & $0.072 \pm 5.1$ & 0.9987 & $83.42 \pm 6.6$ & $0.233 \pm 2.6$ & 0.9985 & $7.358 \pm 3.0$ & $93.11 \pm 5.9$ \\
\hline AAPH & $-77.82 \pm 9.1$ & $0.002 \pm 8.2$ & 0.9991 & $436.3 \pm 4.2$ & $-0.123 \pm 3.6$ & 0.9970 & $-0.178 \pm 3.3$ & $275.18 \pm 7.1$ \\
\hline \multicolumn{9}{|c|}{ OXHLIA ASSAY } \\
\hline Trolox & $100.0 \pm 5.4$ & $0.0276 \pm 20.3$ & 0.9911 & $68.04 \pm 1.2$ & $0.320 \pm 28.2$ & 0.9920 & $2.760 \pm 31.2$ & $102.84 \pm 36.1$ \\
\hline \multicolumn{9}{|c|}{ ORAC ASSAY } \\
\hline Grape seed & $100.0 \pm 10.3$ & $7.522 \pm 55.1$ & 0.9878 & $4.067 \pm 12.2$ & $38.05 \pm 33.1$ & 0.9841 & $752.2 \pm 21.3$ & $19.22 \pm 19.9$ \\
\hline
\end{tabular}




\section{APPENDIX SECTION}

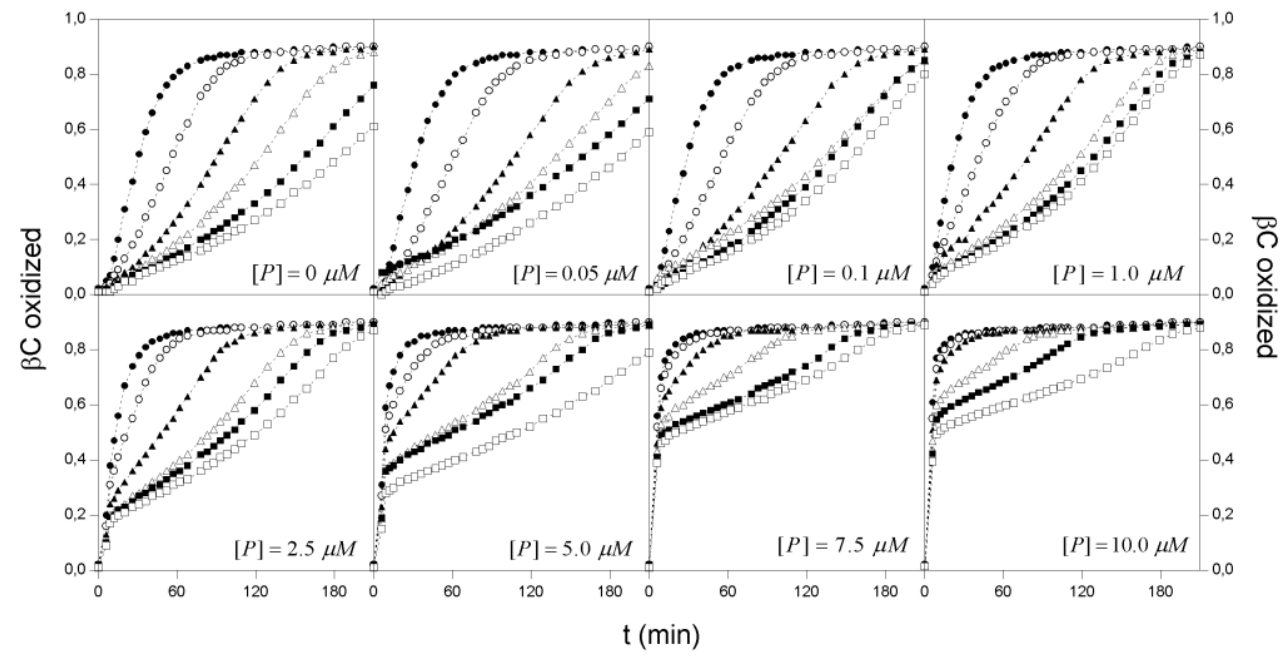

Figure A1: Raw kinetic responses of the combinatory analysis of the antioxidant BHT and the pro-oxidant $\mathrm{Fe}^{+2}$ in the $\beta \mathrm{C}$ assay. Each of the eight dose-response graphs corresponds to a different concentration of $\mathrm{Fe}^{+2}$ at six different concentrations of BHT. 
Table A1: Parametric estimates and confidence intervals $(\alpha=0.05)$ in percentage of the $\beta$ Carotene bleaching kinetics as affected by the specified agents, according to model [2]. All the [A] are in $\mu \mathrm{M}$.

\begin{tabular}{|c|c|c|c|c|c|}
\hline \multirow{2}{*}{ [A] } & \multicolumn{5}{|c|}{ BELL FUNCTION PARAMETERS } \\
\hline & $P_{m}$ & $d$ & $t_{m}$ & $i$ & $\mathbf{r}^{2}$ \\
\hline \multicolumn{6}{|c|}{ BHA } \\
\hline 0.5 & $0.24 \pm 1.9$ & $1.45 \pm 14.4$ & $61.34 \pm 2.6$ & $1.40 \pm 14.5$ & 0.9947 \\
\hline 1.0 & $0.37 \pm 1.5$ & $1.07 \pm 17.6$ & $74.94 \pm 2.5$ & $1.53 \pm 16.2$ & 0.9956 \\
\hline 1.5 & $0.45 \pm 1.0$ & $0.66 \pm 22.3$ & $84.95 \pm 1.9$ & $2.17 \pm 19.8$ & 0.9981 \\
\hline 2.0 & $0.50 \pm 1.2$ & $0.64 \pm 30.1$ & $92.55 \pm 2.3$ & $2.06 \pm 25.6$ & 0.9973 \\
\hline 2.5 & $0.54 \pm 1.2$ & $0.50 \pm 38.9$ & $98.21 \pm 2.2$ & $2.35 \pm 33.0$ & 0.9977 \\
\hline 3.0 & $0.57 \pm 1.0$ & $0.45 \pm 40.3$ & $101.94 \pm 2.0$ & $2.54 \pm 34.3$ & 0.9982 \\
\hline 3.5 & $0.59 \pm 1.3$ & $0.44 \pm 51.7$ & $105.88 \pm 2.5$ & $2.40 \pm 43.2$ & 0.9973 \\
\hline 4.0 & $0.62 \pm 1.2$ & $0.46 \pm 49.7$ & $110.51 \pm 2.4$ & $2.31 \pm 40.6$ & 0.9976 \\
\hline 4.5 & $0.63 \pm 1.3$ & $0.57 \pm 43.6$ & $112.62 \pm 2.6$ & $1.83 \pm 33.4$ & 0.9971 \\
\hline 5.0 & $0.64 \pm 1.2$ & $0.63 \pm 37.6$ & $115.33 \pm 2.5$ & $1.60 \pm 27.5$ & 0.9975 \\
\hline \multicolumn{6}{|c|}{ BHT } \\
\hline 3.0 & $0.31 \pm 1.7$ & $1.63 \pm 11.5$ & $49.98 \pm 2.1$ & $1.44 \pm 11.3$ & 0.9975 \\
\hline 6.0 & $0.40 \pm 1.3$ & $1.68 \pm 8.5$ & $56.20 \pm 1.7$ & $1.27 \pm 8.4$ & 0.9981 \\
\hline 9.0 & $0.49 \pm 1.0$ & $1.81 \pm 6.2$ & $65.27 \pm 1.3$ & $1.21 \pm 6.1$ & 0.9987 \\
\hline 12.0 & $0.57 \pm 0.9$ & $1.72 \pm 6.9$ & $75.83 \pm 1.4$ & $1.16 \pm 6.4$ & 0.9985 \\
\hline 15.0 & $0.62 \pm 1.0$ & $1.57 \pm 9.4$ & $82.38 \pm 1.7$ & $1.15 \pm 8.0$ & 0.9979 \\
\hline 18.0 & $0.64 \pm 1.0$ & $1.15 \pm 13.4$ & $86.64 \pm 1.8$ & $1.37 \pm 11.1$ & 0.9979 \\
\hline 21.0 & $0.67 \pm 0.8$ & $0.88 \pm 14.3$ & $92.08 \pm 1.5$ & $1.48 \pm 11.4$ & 0.9987 \\
\hline 24.0 & $0.69 \pm 0.6$ & $0.63 \pm 15.5$ & $97.21 \pm 1.2$ & $1.77 \pm 12.5$ & 0.9993 \\
\hline 27.0 & $0.70 \pm 0.7$ & $0.48 \pm 23.6$ & $101.72 \pm 1.3$ & $2.10 \pm 19.4$ & 0.9992 \\
\hline 30.0 & $0.70 \pm 0.6$ & $0.37 \pm 28.5$ & $102.35 \pm 1.3$ & $2.62 \pm 24.4$ & 0.9993 \\
\hline \multicolumn{6}{|c|}{ ETX } \\
\hline 0.0004 & $0.08 \pm 4.9$ & $1.21 \pm 33.0$ & $29.03 \pm 7.8$ & $1.10 \pm 36.4$ & 0.9802 \\
\hline 0.0008 & $0.18 \pm 2.9$ & $0.92 \pm 26.6$ & $32.90 \pm 4.1$ & $1.92 \pm 28.2$ & 0.9931 \\
\hline 0.0012 & $0.25 \pm 3.2$ & $0.92 \pm 31.8$ & $37.06 \pm 4.4$ & $2.16 \pm 33.3$ & 0.9917 \\
\hline 0.0016 & $0.37 \pm 2.9$ & $1.14 \pm 27.1$ & $44.53 \pm 3.4$ & $2.30 \pm 27.9$ & 0.9934 \\
\hline 0.0020 & $0.44 \pm 2.2$ & $1.62 \pm 16.6$ & $53.18 \pm 2.5$ & $1.75 \pm 16.4$ & 0.9959 \\
\hline 0.0024 & $0.53 \pm 1.8$ & $1.97 \pm 11.6$ & $64.39 \pm 1.9$ & $1.50 \pm 11.3$ & 0.9967 \\
\hline 0.0028 & $0.57 \pm 0.8$ & $1.87 \pm 5.7$ & $72.90 \pm 1.1$ & $1.34 \pm 5.6$ & 0.9990 \\
\hline 0.0032 & $0.65 \pm 1.4$ & $2.56 \pm 9.0$ & $85.84 \pm 1.9$ & $1.09 \pm 7.8$ & 0.9968 \\
\hline 0.0036 & $0.66 \pm 1.4$ & $2.21 \pm 10.4$ & $91.13 \pm 2.0$ & $1.09 \pm 8.4$ & 0.9968 \\
\hline 0.0040 & $0.66 \pm 2.4$ & $2.36 \pm 19.8$ & $100.54 \pm 3.7$ & $0.98 \pm 14.0$ & 0.9901 \\
\hline \multicolumn{6}{|c|}{ TOC } \\
\hline 0.004 & $0.08 \pm 3.7$ & $1.19 \pm 32.3$ & $52.52 \pm 4.4$ & $2.03 \pm 33.0$ & 0.9853 \\
\hline 0.008 & $0.16 \pm 3.0$ & $0.94 \pm 33.0$ & $56.42 \pm 3.5$ & $2.59 \pm 34.1$ & 0.9895 \\
\hline 0.012 & $0.20 \pm 3.7$ & $1.18 \pm 33.9$ & $62.61 \pm 4.9$ & $1.84 \pm 34.4$ & 0.9805 \\
\hline 0.016 & $0.24 \pm 2.0$ & $0.95 \pm 24.3$ & $67.60 \pm 2.7$ & $2.30 \pm 24.4$ & 0.9939 \\
\hline 0.020 & $0.28 \pm 1.5$ & $0.47 \pm 43.6$ & $74.84 \pm 2.5$ & $3.83 \pm 42.4$ & 0.9959 \\
\hline 0.024 & $0.30 \pm 1.8$ & $0.36 \pm 72.1$ & $79.77 \pm 3.2$ & $4.50 \pm 69.3$ & 0.9941 \\
\hline 0.028 & $0.34 \pm 2.1$ & $0.65 \pm 48.6$ & $88.18 \pm 4.0$ & $2.01 \pm 41.9$ & 0.9915 \\
\hline 0.032 & $0.36 \pm 1.7$ & $0.24 \pm 15.3$ & $92.97 \pm 3.2$ & $5.28 \pm 18.3$ & 0.9952 \\
\hline 0.036 & $0.37 \pm 4.2$ & $0.24 \pm 28.5$ & $92.97 \pm 8.0$ & $5.28 \pm 21.1$ & 0.9891 \\
\hline 0.040 & $0.38 \pm 1.4$ & $0.18 \pm 15.3$ & $102.99 \pm 2.6$ & $6.88 \pm 37.0$ & 0.9969 \\
\hline \multicolumn{6}{|c|}{ PG } \\
\hline 10.0 & $0.27 \pm 1.9$ & $0.86 \pm 20.3$ & $49.89 \pm 2.9$ & $1.93 \pm 21.0$ & 0.9948 \\
\hline 20.0 & $0.32 \pm 1.7$ & $0.60 \pm 28.2$ & $55.16 \pm 3.0$ & $2.37 \pm 28.3$ & 0.9945 \\
\hline 30.0 & $0.31 \pm 1.6$ & $0.68 \pm 24.4$ & $56.51 \pm 3.1$ & $1.90 \pm 24.1$ & 0.9945 \\
\hline 40.0 & $0.37 \pm 1.4$ & $0.39 \pm 40.4$ & $62.82 \pm 3.0$ & $2.79 \pm 38.8$ & 0.9955 \\
\hline 50.0 & $0.37 \pm 1.3$ & $0.27 \pm 58.9$ & $64.90 \pm 2.9$ & $4.08 \pm 57.1$ & 0.9958 \\
\hline 60.0 & $0.38 \pm 1.5$ & $0.38 \pm 47.9$ & $68.48 \pm 3.3$ & $2.70 \pm 45.0$ & 0.9948 \\
\hline 70.0 & $0.43 \pm 1.1$ & $0.43 \pm 34.4$ & $73.65 \pm 2.6$ & $2.36 \pm 31.5$ & 0.9969 \\
\hline 80.0 & $0.42 \pm 1.0$ & $0.21 \pm 66.1$ & $72.84 \pm 2.4$ & $4.74 \pm 63.4$ & 0.9975 \\
\hline 90.0 & $0.47 \pm 1.3$ & $0.29 \pm 61.4$ & $77.83 \pm 3.1$ & $3.05 \pm 56.6$ & 0.9960 \\
\hline 100.0 & $0.49 \pm 1.3$ & $0.01 \pm 33.9$ & $79.39 \pm 3.0$ & $6.55 \pm 29.7$ & 0.9965 \\
\hline
\end{tabular}


Table A2: Parametric estimates and confidence intervals $(\alpha=0.05)$ in percentage of the crocin bleaching kinetics as affected by the specified agents, according to model [2]. All the [P] are in $\mu \mathrm{M}$.

\begin{tabular}{|c|c|c|c|c|c|}
\hline \multirow{2}{*}[\mathbf{P}]{} & \multicolumn{5}{|c|}{ BELL FUNCTION PARAMETERS } \\
\hline & $P_{m}$ & $d$ & $t_{m}$ & $i$ & $\mathbf{r}^{2}$ \\
\hline \multicolumn{6}{|c|}{$\beta$-Carotene reaction } \\
\hline \multicolumn{6}{|c|}{$\mathrm{Fe}^{+2}$} \\
\hline 1.5 & $-0.25 \pm 1.6$ & $1.76 \pm 8.1$ & $21.98 \pm 3.2$ & $0.42 \pm 11.6$ & 0.9983 \\
\hline 3.0 & $-0.28 \pm 16.1$ & $1.77 \pm 57.9$ & $21.81 \pm 22.1$ & $0.43 \pm 93.4$ & 0.9981 \\
\hline 4.5 & $-0.36 \pm 11.1$ & $1.57 \pm 55.1$ & $18.09 \pm 22.8$ & $0.39 \pm 75.1$ & 0.9989 \\
\hline 6.0 & $-0.49 \pm 1.2$ & $1.33 \pm 6.6$ & $14.06 \pm 2.9$ & $0.38 \pm 11.1$ & 0.9992 \\
\hline 7.5 & $-0.54 \pm 1.7$ & $1.20 \pm 9.9$ & $12.33 \pm 4.1$ & $0.40 \pm 17.0$ & 0.9983 \\
\hline 9.0 & $-0.57 \pm 1.3$ & $1.18 \pm 7.6$ & $11.77 \pm 3.5$ & $0.37 \pm 13.4$ & 0.9990 \\
\hline 10.5 & $-0.59 \pm 1.4$ & $1.15 \pm 8.2$ & $11.19 \pm 3.9$ & $0.37 \pm 14.6$ & 0.9989 \\
\hline 12.0 & $-0.61 \pm 1.4$ & $1.10 \pm 8.5$ & $10.65 \pm 4.1$ & $0.38 \pm 15.2$ & 0.9988 \\
\hline 13.5 & $-0.63 \pm 1.7$ & $1.05 \pm 11.3$ & $9.72 \pm 5.0$ & $0.42 \pm 20.2$ & 0.9983 \\
\hline 15.0 & $-0.65 \pm 1.5$ & $1.02 \pm 9.0$ & $9.91 \pm 4.3$ & $0.39 \pm 16.3$ & 0.9987 \\
\hline \multicolumn{6}{|c|}{$\mathrm{Cu}^{+2}$} \\
\hline 15.0 & $-0.12 \pm 3.4$ & $0.92 \pm 29.4$ & $39.12 \pm 6.1$ & $1.25 \pm 31.3$ & 0.9840 \\
\hline 30.0 & $-0.20 \pm 2.5$ & $1.06 \pm 19.3$ & $36.68 \pm 4.4$ & $1.11 \pm 20.7$ & 0.9922 \\
\hline 60.0 & $-0.28 \pm 2.0$ & $0.96 \pm 16.1$ & $32.01 \pm 3.6$ & $1.16 \pm 17.7$ & 0.9955 \\
\hline 90.0 & $-0.32 \pm 2.0$ & $0.89 \pm 16.6$ & $29.67 \pm 3.6$ & $1.21 \pm 18.5$ & 0.9958 \\
\hline 120.0 & $-0.35 \pm 1.9$ & $0.86 \pm 15.6$ & $28.12 \pm 3.3$ & $1.23 \pm 17.6$ & 0.9965 \\
\hline 150.0 & $-0.36 \pm 2.0$ & $0.81 \pm 17.2$ & $26.80 \pm 3.5$ & $1.27 \pm 19.4$ & 0.9962 \\
\hline 180.0 & $-0.38 \pm 1.5$ & $0.83 \pm 12.4$ & $26.58 \pm 2.6$ & $1.22 \pm 14.1$ & 0.9979 \\
\hline 210.0 & $-0.39 \pm 1.2$ & $0.73 \pm 11.1$ & $25.38 \pm 2.1$ & $1.36 \pm 12.6$ & 0.9986 \\
\hline 240.0 & $-0.40 \pm 1.5$ & $0.75 \pm 13.6$ & $25.18 \pm 2.7$ & $1.32 \pm 15.5$ & 0.9979 \\
\hline \multicolumn{6}{|c|}{$\mathbf{H b}$} \\
\hline 0.2 & $-0.06- \pm 25.8$ & $0.05 \pm 41.4$ & $32.40 \pm 39.1$ & $2.27 \pm 47.1$ & 0.8617 \\
\hline 2.0 & $-0.33- \pm 4.5$ & $0.80 \pm 38.6$ & $25.35 \pm 7.8$ & $1.27 \pm 44.0$ & 0.9821 \\
\hline 4.0 & $-0.46- \pm 3.1$ & $0.64 \pm 29.7$ & $18.39 \pm 5.5$ & $1.28 \pm 35.5$ & 0.9927 \\
\hline 6.0 & $-0.54- \pm 2.8$ & $0.71 \pm 24.4$ & $14.62 \pm 5.5$ & $0.88 \pm 32.7$ & 0.9947 \\
\hline 8.0 & $-0.59- \pm 2.7$ & $0.65 \pm 25.9$ & $12.49 \pm 5.7$ & $0.85 \pm 36.0$ & 0.9952 \\
\hline 10.0 & $-0.62- \pm 2.7$ & $0.66 \pm 25.7$ & $10.98 \pm 6.3$ & $0.72 \pm 38.3$ & 0.9954 \\
\hline 12.0 & $-0.65- \pm 2.4$ & $0.66 \pm 22.9$ & $9.72 \pm 6.2$ & $0.64 \pm 36.3$ & 0.9965 \\
\hline 16.0 & $-0.67- \pm 2.6$ & $0.71 \pm 22.1$ & $8.65 \pm 8.4$ & $0.51 \pm 38.5$ & 0.9957 \\
\hline 20.0 & $-0.68- \pm 2.5$ & $0.66 \pm 21.6$ & $7.93 \pm 8.7$ & $0.51 \pm 38.0$ & 0.9961 \\
\hline \multicolumn{6}{|c|}{ Crocin reaction } \\
\hline \multicolumn{6}{|c|}{ AАPH } \\
\hline 200.0 & $-30.8 \pm 0.2$ & $1.26 \pm 1.5$ & $533.53 \pm 0.2$ & $1.63 \pm 1.7$ & 1.0000 \\
\hline 400.0 & $-47.1 \pm 0.3$ & $0.96 \pm 2.9$ & $428.75 \pm 0.4$ & $1.86 \pm 3.3$ & 0.9999 \\
\hline 600.0 & $-57.1 \pm 0.4$ & $0.76 \pm 4.0$ & $365.61 \pm 0.5$ & $2.08 \pm 4.6$ & 0.9999 \\
\hline 800.0 & $-63.9 \pm 0.6$ & $0.64 \pm 6.5$ & $323.56 \pm 0.8$ & $2.24 \pm 7.4$ & 0.9997 \\
\hline 1000.0 & $-68.7 \pm 0.8$ & $0.56 \pm 10.5$ & $293.59 \pm 1.2$ & $2.34 \pm 11.9$ & 0.9995 \\
\hline 1200.0 & $-72.4 \pm 1.1$ & $0.52 \pm 14.9$ & $271.20 \pm 1.7$ & $2.36 \pm 17.0$ & 0.9991 \\
\hline 1400.0 & $-75.2 \pm 1.4$ & $0.49 \pm 19.2$ & $253.88 \pm 2.2$ & $2.31 \pm 22.0$ & 0.9986 \\
\hline 1600.0 & $-77.3 \pm 1.7$ & $0.49 \pm 22.7$ & $240.11 \pm 2.6$ & $2.19 \pm 26.4$ & 0.9981 \\
\hline 1800.0 & $-79.1 \pm 1.9$ & $0.49 \pm 25.3$ & $228.91 \pm 3.0$ & $2.03 \pm 29.9$ & 0.9976 \\
\hline 2000.0 & $-80.4 \pm 2.1$ & $0.51 \pm 26.8$ & $219.58 \pm 3.4$ & $1.85 \pm 32.2$ & 0.9972 \\
\hline
\end{tabular}


Table A3: Parametric estimates and confidence intervals $(\alpha=0.05)$ in percentage of the crocin bleaching kinetics as affected by the specified agents, according to model [2]. All the [A] are in $\mu \mathrm{M}$.

\begin{tabular}{|c|c|c|c|c|c|}
\hline \multirow{2}{*}[\mathbf{A}]{} & \multicolumn{5}{|c|}{ BELL FUNCTION PARAMETERS } \\
\hline & $\boldsymbol{P}_{\boldsymbol{m}}$ & $d$ & $t_{m}$ & $i$ & $\mathbf{r}^{2}$ \\
\hline \multicolumn{6}{|c|}{ AA } \\
\hline 30.0 & $39.74 \pm 1.8$ & $1.60 \pm 11.7$ & $32.82 \pm 2.2$ & $1.37 \pm 11.7$ & 0.9977 \\
\hline 60.0 & $58.75 \pm 1.6$ & $1.85 \pm 9.3$ & $44.77 \pm 1.9$ & $1.25 \pm 9.1$ & 0.9978 \\
\hline 90.0 & $65.31 \pm 1.4$ & $1.96 \pm 8.1$ & $49.50 \pm 1.7$ & $1.18 \pm 7.7$ & 0.9982 \\
\hline 120.0 & $68.21 \pm 1.1$ & $2.07 \pm 6.3$ & $52.54 \pm 1.4$ & $1.10 \pm 5.9$ & 0.9987 \\
\hline 150.0 & $69.80 \pm 1.0$ & $2.06 \pm 5.8$ & $54.33 \pm 1.3$ & $1.10 \pm 5.4$ & 0.9989 \\
\hline 180.0 & $75.45 \pm 1.2$ & $2.42 \pm 6.7$ & $61.41 \pm 1.6$ & $0.96 \pm 5.9$ & 0.9982 \\
\hline 210.0 & $74.75 \pm 1.2$ & $2.33 \pm 7.0$ & $61.17 \pm 1.7$ & $0.98 \pm 6.2$ & 0.9981 \\
\hline 240.0 & $76.89 \pm 1.3$ & $2.42 \pm 7.4$ & $64.45 \pm 1.8$ & $0.93 \pm 6.4$ & 0.9977 \\
\hline 270.0 & $78.30 \pm 1.4$ & $2.32 \pm 7.7$ & $65.79 \pm 1.9$ & $0.96 \pm 6.7$ & 0.9975 \\
\hline 300.0 & $79.21 \pm 1.6$ & $2.46 \pm 8.9$ & $68.56 \pm 2.2$ & $0.89 \pm 7.5$ & 0.9963 \\
\hline \multicolumn{6}{|c|}{ ETX } \\
\hline 3.0 & $49.56 \pm 2.6$ & $1.91 \pm 15.9$ & $36.20 \pm 2.8$ & $1.44 \pm 15.3$ & 0.9958 \\
\hline 6.0 & $67.86 \pm 1.5$ & $2.06 \pm 9.1$ & $44.16 \pm 1.7$ & $1.36 \pm 8.6$ & 0.9983 \\
\hline 9.0 & $80.45 \pm 1.5$ & $2.36 \pm 8.5$ & $51.57 \pm 1.8$ & $1.22 \pm 7.7$ & 0.9981 \\
\hline 12.0 & $89.36 \pm 1.4$ & $2.51 \pm 7.7$ & $58.26 \pm 1.7$ & $1.13 \pm 6.7$ & 0.9982 \\
\hline 15.0 & $96.81 \pm 1.4$ & $2.61 \pm 7.8$ & $65.42 \pm 1.8$ & $1.04 \pm 6.6$ & 0.9976 \\
\hline 18.0 & $101.15 \pm 1.5$ & $2.63 \pm 8.3$ & $70.15 \pm 1.9$ & $0.98 \pm 6.9$ & 0.9970 \\
\hline 21.0 & $107.01 \pm 1.8$ & $2.64 \pm 9.9$ & $78.67 \pm 2.4$ & $0.91 \pm 8.0$ & 0.9953 \\
\hline 24.0 & $109.60 \pm 2.1$ & $2.62 \pm 12.0$ & $83.55 \pm 2.9$ & $0.86 \pm 9.4$ & 0.9931 \\
\hline 27.0 & $111.12 \pm 2.2$ & $2.58 \pm 13.7$ & $86.60 \pm 3.3$ & $0.85 \pm 10.4$ & 0.9915 \\
\hline 30.0 & $112.40 \pm 2.3$ & $2.57 \pm 14.6$ & $87.92 \pm 3.5$ & $0.83 \pm 10.9$ & 0.9906 \\
\hline \multicolumn{6}{|c|}{ TROLOX } \\
\hline 18.8 & $35.12 \pm 1.0$ & $1.64 \pm 6.6$ & $52.72 \pm 1.2$ & $1.46 \pm 6.4$ & 0.9990 \\
\hline 37.5 & $50.58 \pm 0.5$ & $1.42 \pm 3.7$ & $58.65 \pm 0.8$ & $1.27 \pm 3.6$ & 0.9996 \\
\hline 56.3 & $62.98 \pm 0.8$ & $1.09 \pm 8.4$ & $64.22 \pm 1.4$ & $1.38 \pm 7.7$ & 0.9988 \\
\hline 75.0 & $71.96 \pm 0.8$ & $0.82 \pm 11.1$ & $69.48 \pm 1.4$ & $1.64 \pm 10.0$ & 0.9988 \\
\hline 93.8 & $79.09 \pm 0.9$ & $0.67 \pm 17.0$ & $74.20 \pm 1.8$ & $1.82 \pm 14.9$ & 0.9984 \\
\hline 112.5 & $82.57 \pm 1.0$ & $0.60 \pm 21.0$ & $76.71 \pm 2.0$ & $1.91 \pm 18.3$ & 0.9982 \\
\hline 131.3 & $85.83 \pm 0.9$ & $0.50 \pm 24.9$ & $80.40 \pm 1.9$ & $2.15 \pm 21.7$ & 0.9984 \\
\hline 150.0 & $89.52 \pm 1.0$ & $0.40 \pm 34.1$ & $83.91 \pm 2.0$ & $2.50 \pm 30.0$ & 0.9983 \\
\hline \multicolumn{6}{|c|}{ TBHQ } \\
\hline 80.0 & $24.91 \pm 4.0$ & $2.22 \pm 22.7$ & $54.80 \pm 5.2$ & $1.07 \pm 20.7$ & 0.9831 \\
\hline 160.0 & $29.68 \pm 3.2$ & $1.98 \pm 19.0$ & $58.50 \pm 4.4$ & $1.07 \pm 17.6$ & 0.9870 \\
\hline 240.0 & $34.27 \pm 3.2$ & $1.71 \pm 20.9$ & $60.07 \pm 4.6$ & $1.16 \pm 19.6$ & 0.9855 \\
\hline 320.0 & $37.58 \pm 2.7$ & $1.30 \pm 21.5$ & $60.48 \pm 3.9$ & $1.47 \pm 20.6$ & 0.9894 \\
\hline 400.0 & $41.46 \pm 2.2$ & $1.31 \pm 17.8$ & $61.94 \pm 3.4$ & $1.35 \pm 16.8$ & 0.9921 \\
\hline 480.0 & $45.75 \pm 1.9$ & $1.27 \pm 16.7$ & $63.73 \pm 3.1$ & $1.31 \pm 15.5$ & 0.9933 \\
\hline 560.0 & $49.18 \pm 2.1$ & $1.12 \pm 20.4$ & $64.88 \pm 3.5$ & $1.42 \pm 18.9$ & 0.9919 \\
\hline 640.0 & $51.52 \pm 1.8$ & $1.18 \pm 17.3$ & $66.18 \pm 3.1$ & $1.29 \pm 15.7$ & 0.9935 \\
\hline 720.0 & $50.87 \pm 2.0$ & $0.98 \pm 22.9$ & $67.00 \pm 3.4$ & $1.63 \pm 21.1$ & 0.9927 \\
\hline 800.0 & $50.96 \pm 2.0$ & $1.17 \pm 18.9$ & $65.09 \pm 3.4$ & $1.30 \pm 17.3$ & 0.9922 \\
\hline \multicolumn{6}{|c|}{$\mathrm{Mn}^{+2}$} \\
\hline 12.5 & $66.30 \pm 1.0$ & $0.85 \pm 15.4$ & $78.43 \pm 1.8$ & $1.64 \pm 13.1$ & 0.9981 \\
\hline 25.0 & $82.33 \pm 1.1$ & $0.76 \pm 21.6$ & $88.40 \pm 2.1$ & $1.61 \pm 17.2$ & 0.9978 \\
\hline 37.5 & $92.88 \pm 1.1$ & $0.65 \pm 26.4$ & $93.58 \pm 2.1$ & $1.74 \pm 20.9$ & 0.9979 \\
\hline $\mathbf{5 0 . 0}$ & $94.76 \pm 0.7$ & $0.51 \pm 22.3$ & $97.93 \pm 1.4$ & $1.79 \pm 18.1$ & 0.9992 \\
\hline 62.5 & $99.71 \pm 0.8$ & $0.60 \pm 21.8$ & $100.81 \pm 1.5$ & $1.74 \pm 16.9$ & 0.9990 \\
\hline 75.0 & $101.64 \pm 0.8$ & $0.54 \pm 23.2$ & $102.63 \pm 1.5$ & $1.86 \pm 18.1$ & 0.9991 \\
\hline 87.5 & $104.17 \pm 0.8$ & $0.60 \pm 22.9$ & $104.04 \pm 1.6$ & $1.69 \pm 17.3$ & 0.9990 \\
\hline 100.0 & $106.05 \pm 0.9$ & $0.61 \pm 24.3$ & $105.18 \pm 1.7$ & $1.63 \pm 18.2$ & 0.9988 \\
\hline 112.5 & $105.36 \pm 0.7$ & $0.59 \pm 21.4$ & $105.69 \pm 1.4$ & $1.69 \pm 16.1$ & 0.9992 \\
\hline
\end{tabular}


Table A4: Parametric estimates and confidence intervals $(\alpha=0.05)$ in percentage of the $\beta$ Carotene bleaching kinetics as affected by the specified agents, according to model [2]. All the $[\mathrm{OM}]$ are in $\mu \mathrm{M}$.

\begin{tabular}{|c|c|c|c|c|c|}
\hline \multirow{2}{*}[\mathbf{A}]{} & \multicolumn{5}{|c|}{ BELL FUNCTION PARAMETERS } \\
\hline & $\boldsymbol{P}_{m}$ & $d$ & $t_{m}$ & $\boldsymbol{i}$ & $\mathbf{r}^{2}$ \\
\hline \multicolumn{6}{|c|}{$[\mathrm{P}]=0 \mu \mathrm{M}$} \\
\hline 6.0 & $0.33 \pm 32.9$ & $1.49 \pm 4.5$ & $43.8 \pm 7.3$ & $3.30 \pm 3.4$ & 0.9994 \\
\hline 12.0 & $0.54 \pm 27.1$ & $1.50 \pm 5.5$ & $53.4 \pm 9.8$ & $2.81 \pm 2.8$ & 0.9977 \\
\hline 18.0 & $0.63 \pm 21.1$ & $1.32 \pm 6.2$ & $61.8 \pm 10.1$ & $2.68 \pm 2.1$ & 0.9930 \\
\hline 24.0 & $0.68 \pm 16.9$ & $0.69 \pm 4.1$ & $69.3 \pm 16.6$ & $3.36 \pm 1.0$ & 0.9933 \\
\hline 30.0 & $0.70 \pm 14.0$ & $0.31 \pm 2.2$ & $75.6 \pm 32.0$ & $5.22 \pm 0.4$ & 0.9975 \\
\hline \multicolumn{6}{|c|}{$[\mathrm{P}]=0.05 \mu \mathrm{M}$} \\
\hline 6.0 & $0.34 \pm 34.4$ & $1.42 \pm 4.1$ & $45.0 \pm 8.3$ & $3.40 \pm 3.2$ & 0.9986 \\
\hline 12.0 & $0.57 \pm 28.6$ & $1.35 \pm 4.7$ & $56.6 \pm 12.2$ & $2.92 \pm 2.4$ & 0.9948 \\
\hline 18.0 & $0.62 \pm 20.8$ & $0.79 \pm 3.8$ & $66.0 \pm 16.4$ & $3.61 \pm 1.2$ & 0.9896 \\
\hline 24.0 & $0.62 \pm 15.6$ & $0.11 \pm 0.7$ & $74.3 \pm 87.8$ & $16.64 \pm 0.1$ & 0.9907 \\
\hline 30.0 & $0.70 \pm 14.1$ & $0.16 \pm 1.2$ & $77.3 \pm 59.9$ & $9.06 \pm 0.2$ & 0.9977 \\
\hline \multicolumn{6}{|c|}{$[\mathrm{P}]=0.1 \mu \mathrm{M}$} \\
\hline 6.0 & $0.34 \pm 33.5$ & $1.45 \pm 4.3$ & $44.0 \pm 7.7$ & $3.19 \pm 3.3$ & 0.9993 \\
\hline 12.0 & $0.56 \pm 28.0$ & $1.43 \pm 5.1$ & $53.8 \pm 11.0$ & $2.65 \pm 2.7$ & 0.9960 \\
\hline 18.0 & $0.61 \pm 20.4$ & $0.83 \pm 4.1$ & $63.2 \pm 15.0$ & $3.34 \pm 1.3$ & 0.9870 \\
\hline 24.0 & $0.66 \pm 16.4$ & $1.14 \pm 6.9$ & $68.6 \pm 9.5$ & $2.61 \pm 1.7$ & 0.9891 \\
\hline 30.0 & $0.68 \pm 13.5$ & $0.88 \pm 6.5$ & $75.9 \pm 10.4$ & $2.89 \pm 1.2$ & 0.9883 \\
\hline \multicolumn{6}{|c|}{$[\mathrm{P}]=1.0 \mu \mathrm{M}$} \\
\hline 6.0 & $0.28 \pm 27.6$ & $1.29 \pm 4.7$ & $37.7 \pm 5.9$ & $3.24 \pm 3.4$ & 0.9996 \\
\hline 12.0 & $0.50 \pm 25.0$ & $1.36 \pm 5.4$ & $49.2 \pm 9.2$ & $2.63 \pm 2.8$ & 0.9949 \\
\hline 18.0 & $0.62 \pm 20.5$ & $1.39 \pm 6.8$ & $57.8 \pm 9.1$ & $2.27 \pm 2.4$ & 0.9880 \\
\hline 24.0 & $0.65 \pm 16.2$ & $1.35 \pm 8.3$ & $64.2 \pm 7.7$ & $2.21 \pm 2.1$ & 0.9849 \\
\hline 30.0 & $0.66 \pm 13.2$ & $1.29 \pm 9.8$ & $70.2 \pm 6.7$ & $2.20 \pm 1.8$ & 0.9828 \\
\hline \multicolumn{6}{|c|}{$[\mathrm{P}]=2.5 \mu \mathrm{M}$} \\
\hline 6.0 & $0.18 \pm 18.0$ & $1.21 \pm 6.7$ & $27.0 \pm 2.7$ & $3.25 \pm 4.5$ & 0.9993 \\
\hline 12.0 & $0.38 \pm 19.2$ & $1.37 \pm 7.1$ & $43.0 \pm 5.4$ & $2.36 \pm 3.2$ & 0.9969 \\
\hline 18.0 & $0.51 \pm 17.1$ & $1.48 \pm 8.7$ & $50.8 \pm 5.9$ & $1.90 \pm 2.9$ & 0.9867 \\
\hline 24.0 & $0.53 \pm 13.3$ & $1.40 \pm 10.6$ & $57.9 \pm 5.0$ & $1.87 \pm 2.4$ & 0.9804 \\
\hline 30.0 & $0.59 \pm 11.8$ & $1.18 \pm 10.0$ & $65.8 \pm 5.9$ & $1.90 \pm 1.8$ & 0.9735 \\
\hline \multicolumn{6}{|c|}{$[\mathrm{P}]=5.0 \mu \mathrm{M}$} \\
\hline 6.0 & $0.13 \pm 12.9$ & $0.43 \pm 3.4$ & $21.2 \pm 3.8$ & $5.80 \pm 2.0$ & 0.9930 \\
\hline 12.0 & $0.23 \pm 11.7$ & $1.06 \pm 9.0$ & $31.6 \pm 2.6$ & $2.53 \pm 3.3$ & 0.9962 \\
\hline 18.0 & $0.39 \pm 12.9$ & $1.36 \pm 10.6$ & $42.8 \pm 3.7$ & $1.67 \pm 3.2$ & 0.9796 \\
\hline 24.0 & $0.40 \pm 10.0$ & $1.31 \pm 13.1$ & $50.1 \pm 3.0$ & $1.63 \pm 2.6$ & 0.9730 \\
\hline 30.0 & $0.49 \pm 9.7$ & $0.62 \pm 6.4$ & $57.3 \pm 7.6$ & $1.98 \pm 1.1$ & 0.9661 \\
\hline \multicolumn{6}{|c|}{$[\mathrm{P}]=7.5 \mu \mathrm{M}$} \\
\hline 6.0 & $0.05 \pm 5.0$ & $1.56 \pm 31.2$ & $14.3 \pm 0.2$ & $1.84 \pm 10.8$ & 0.9859 \\
\hline 12.0 & $0.15 \pm 7.6$ & $1.30 \pm 17.0$ & $21.9 \pm 0.9$ & $1.86 \pm 5.9$ & 0.9960 \\
\hline 18.0 & $0.23 \pm 7.7$ & $1.43 \pm 18.6$ & $32.7 \pm 1.2$ & $1.62 \pm 4.4$ & 0.9933 \\
\hline 24.0 & $0.30 \pm 7.4$ & $1.72 \pm 23.3$ & $41.8 \pm 1.3$ & $1.36 \pm 4.1$ & 0.9824 \\
\hline 30.0 & $0.36 \pm 7.2$ & $1.76 \pm 24.3$ & $49.7 \pm 1.5$ & $1.31 \pm 3.5$ & 0.9762 \\
\hline \multicolumn{6}{|c|}{$[P]=10.0 \mu \mathrm{M}$} \\
\hline 6.0 & $0.04 \pm 4.3$ & $0.22 \pm 5.1$ & $7.7 \pm 0.8$ & $3.77 \pm 2.9$ & 0.9888 \\
\hline 12.0 & $0.12 \pm 5.9$ & $0.27 \pm 4.6$ & $12.0 \pm 2.6$ & $3.96 \pm 2.2$ & 0.9868 \\
\hline 18.0 & $0.18 \pm 6.0$ & $1.69 \pm 28.3$ & $19.5 \pm 0.6$ & $1.37 \pm 8.7$ & 0.9966 \\
\hline 24.0 & $0.21 \pm 5.2$ & $1.68 \pm 32.3$ & $29.6 \pm 0.6$ & $1.26 \pm 5.7$ & 0.9927 \\
\hline 30.0 & $0.27 \pm 5.5$ & $1.65 \pm 30.3$ & $38.0 \pm 0.9$ & $1.21 \pm 4.4$ & 0.9788 \\
\hline
\end{tabular}


Table A5: Parametric estimates and confidence intervals $(\alpha=0.05)$ in percentage of the crocin bleaching kinetics as affected by the specified agents, according to model [2]. All the [A] are in $\mu \mathrm{M}$.

\begin{tabular}{|c|c|c|c|c|c|}
\hline \multirow{2}{*}[\mathbf{A}]{} & \multicolumn{5}{|c|}{ BELL FUNCTION PARAMETERS } \\
\hline & $\boldsymbol{P}_{m}$ & $d$ & $t_{m}$ & $i$ & $\mathbf{r}^{2}$ \\
\hline \multicolumn{6}{|c|}{ OxHLIA ASSAY } \\
\hline 25.0 & $42.11 \pm 2.1$ & $1.36 \pm 16.9$ & $73.37 \pm 2.4$ & $14.33 \pm 1.6$ & 0.9980 \\
\hline 50.0 & $73.57 \pm 1.4$ & $2.85 \pm 7.3$ & $84.25 \pm 2.5$ & $7.01 \pm 3.9$ & 0.9991 \\
\hline 75.0 & $90.82 \pm 2.1$ & $4.25 \pm 3.8$ & $93.62 \pm 1.9$ & $4.37 \pm 10.8$ & 0.9977 \\
\hline 100.0 & $98.92 \pm 3.0$ & $3.89 \pm 5.4$ & $100.40 \pm 2.3$ & $3.87 \pm 15.0$ & 0.9948 \\
\hline 125.0 & $99.00 \pm 3.6$ & $3.49 \pm 6.2$ & $106.92 \pm 2.2$ & $3.35 \pm 25.5$ & 0.9920 \\
\hline \multicolumn{6}{|c|}{$O R A C A S S A Y$} \\
\hline 0.05 & $30.71 \pm 1.6$ & $0.95 \pm 17.1$ & $5.81 \pm 1.6$ & $3.15 \pm 18.6$ & 0.9984 \\
\hline 0.10 & $52.16 \pm 1.3$ & $1.51 \pm 9.9$ & $8.03 \pm 1.4$ & $1.98 \pm 10.7$ & 0.9986 \\
\hline 0.20 & $78.49 \pm 1.9$ & $1.89 \pm 13.3$ & $11.62 \pm 2.5$ & $1.26 \pm 13.7$ & 0.9946 \\
\hline
\end{tabular}




\section{REFERENCES}

Balk, J. M., Bast, A., \& Haenen, G. R. M. M. (2009). Evaluation of the accuracy of antioxidant competition assays: incorrect assumptions with major impact. Free Radical Biology and Medicine, 47(2), 135-144.

Carocho, M., \& Ferreira, I. C. F. R. (2013). A review on antioxidants, prooxidants and related controversy: Natural and synthetic compounds, screening and analysis methodologies and future perspectives. Food and Chemical Toxicology, 51(0), 15-25.

Comuzzi, C., Polese, P., Melchior, A., Portanova, R., \& Tolazzi, M. (2003). SOLVERSTAT: a new utility for multipurpose analysis. An application to the investigation of dioxygenated Co(II) complex formation in dimethylsulfoxide solution. Talanta, 59(1), 67-80.

Chatterjee, S., Poduval, T. B., Tilak, J. C., \& Devasagayam, T. P. A. (2005). A modified, economic, sensitive method for measuring total antioxidant capacities of human plasma and natural compounds using Indian saffron (Crocus sativus). Clinica Chimica Acta, 352(1-2), 155163.

Di Marco. V., \& Bombi, G. G. (2001). Mathematical functions for the representation of chromatographic peaks. Journal of chromatography.A, 931(1-2), 1-30.

Dawidowicz, A. L., \& Olszowy, M. (2010). Influence of some experimental variables and matrix components in the determination of antioxidant properties by $\beta$-carotene bleaching assay: Experiments with BHT used as standard antioxidant. European Food Research and Technology, 231(6), 835-840.

Frankel, E. N. (1994). Methods of evaluating food antioxidants: reply. Trends in Food Science and Technology, 5(2), 57.

Frankel, E. N. (1993). In search of better methods to evaluate natural antioxidants and oxidative stability in food lipids. Trends in Food Science \& Technology, 4(7), 220-225.

Frankel, E. N., \& Finley, J. W. (2008). How to standardize the multiplicity of methods to evaluate natural antioxidants. Journal of Agricultural and Food Chemistry, 56(13), 4901-4908.

Frankel, E. N., \& Meyer, A. S. (2000). The problems of using one-dimensional methods to evaluate multifunctional food and biological antioxidants. Journal of the science of food and agriculture, 80(13), 1925-1941.

Gieseg, S. P., \& Esterbauer, H. (1994). Low density lipoprotein is saturable by pro-oxidant copper. FEBS letters, 343(3), 188-194.

Gutteridge, J. M. C., \& Halliwell, B. (2010). Antioxidants: Molecules, medicines, and myths. Biochemical and biophysical research communications, 393(4), 561-564.

Halliwell, B. (2013). The antioxidant paradox: Less paradoxical now? British journal of clinical pharmacology, 75(3), 637-644. 
Halliwell, B. (2012). Free radicals and antioxidants: Updating a personal view. Nutrition reviews, 70(5), 257-265.

Huang, D., Boxin, O. U., \& Prior, R. L. (2005). The chemistry behind antioxidant capacity assays. Journal of Agricultural and Food Chemistry, 53(6), 1841-1856.

Hussain, S. P., Hofseth, L. J., \& Harris, C. C. (2003). Radical causes of cancer. Nature reviews.Cancer, 3(4), 276-285.

Kalyanaraman, B. (2004). Introduction to the Review Series on redox-active metal ions, reactive oxygen species and apoptosis. Free Radical Biology and Medicine, 37(5), 573.

Koleva, I. I., Van Beek, T. A., Linssen, J. P. H., De Groot, A., \& Evstatieva, L. N. (2002). Screening of plant extracts for antioxidant activity: A comparative study on three testing methods. Phytochemical Analysis, 13(1), 8-17.

Labuza, T. P., \& Dugan, L. R. (1971). Kinetics of lipid oxidation in foods. CRC Critical Reviews in Food Technology, 2(3), 355.

Laguerre, M., Lecomte, J., \& Villeneuve, P. (2007). Evaluation of the ability of antioxidants to counteract lipid oxidation: Existing methods, new trends and challenges. Progress in lipid research, 46(5), 244-282.

Murado, M. A., \& Vázquez, J. A. (2010). Mathematical model for the characterization and objective comparison of antioxidant activities. Journal of Agricultural and Food Chemistry, $58(3), 1622-1629$.

Naguib, Y. M. A. (2000). A Fluorometric Method for Measurement of Oxygen RadicalScavenging Activity of Water-Soluble Antioxidants. Analytical Biochemistry, 284(1), 93-98.

Notas, G., Miliaraki, N., Kampa, M., Dimoulios, F., Matrella, E., Hatzidakis, A., Castanas, E., \& Kouroumalis, E. (2005). Patients with primary biliary cirrhosis have increased serum total antioxidant capacity measured with the crocin bleaching assay. World journal of gastroenterology, 11(27), 4194-4198.

Ordoudi, S. A., \& Tsimidou, M. Z. (2006). Crocin bleaching assay step by step: observations and suggestions for an alternative validated protocol. Journal of Agricultural and Food Chemistry, 54(5), 1663-1671.

Ou, B., Hampsch-Woodill, M., \& Prior, R. L. (2001). Development and validation of an improved oxygen radical absorbance capacity assay using fluorescein as the fluorescent probe. Journal of Agricultural and Food Chemistry, 49(10), 4619-4626.

Özilgen, S., \& Özilgen, M. (1990). Kinetic Model of Lipid Oxidation in Foods. Journal of Food Science, 55(2), 498-498.

Prieto, M. A., Murado, M. A., Vázquez, J. A., Anders, Y., \& Curran, T. P. (2013a). A new microplate procedure for simultaneous assessment of lipophilic and hydrophilic antioxidants and pro-oxidants, using crocin and $\beta$-carotene bleaching methods in a single combined assay: Tea extracts as a case study. Food Research International, 53(2), 836-846. 
Prieto, M. A., Murado, M. A., \& Vázquez, J. A. (2013b). Quantification, characterization and description of synergy and antagonism in the antioxidant response. Food Research International, (in press).

Prieto, M. A., Rodríguez-Amado, I., Vázquez, J. A., \& Murado, M. A. (2012). $\beta$-Carotene Assay Revisited. Application To Characterize and Quantify Antioxidant and Prooxidant Activities in a Microplate. Journal of Agricultural and Food Chemistry, .

Prieto, M. A., Vázquez, J. A., \& Murado, M. A. (2011). Hydrolysis optimization of mannan, curdlan and cell walls from Endomyces fibuliger grown in mussel processing wastewaters. Process Biochemistry, 46(8), 1579-1588.

Prikler, S. (2009). Robert de Levie: Advanced Excel for scientific data analysis, 2nd ed. Analytical and Bioanalytical Chemistry, 395(7), 1945.

Prior, R. L., Wu, X., \& Schaich, K. (2005). Standardized methods for the determination of antioxidant capacity and phenolics in foods and dietary supplements. Journal of Agricultural and Food Chemistry, 53(10), 4290-4302.

Prior, R. L., \& Cao, G. (1999). In vivo total antioxidant capacity: comparison of different analytical methods1. Free Radical Biology and Medicine, 27(11-12), 1173-1181.

Roginsky, V., \& Lissi, E. A. (2005). Review of methods to determine chain-breaking antioxidant activity in food. Food Chemistry, 92(2), 235-254.

Takebayashi, J., Chen, J., \& Tai, A. (2010). A method for evaluation of antioxidant activity based on inhibition of free radical-induced erythrocyte hemolysis. Methods in molecular biology, 594, 287-296.

Terpinc, P., \& Abramovič, H. (2010). A kinetic approach for evaluation of the antioxidant activity of selected phenolic acids. Food Chemistry, 121(2), 366-371.

Tsuchihashi, H., Kigoshi, M., Iwatsuki, M., \& Niki, E. (1995). Action of $\beta$-carotene as an antioxidant against lipid peroxidation. Archives of Biochemistry and Biophysics, 323(1), 137147.

Wardhani, D. H., Fuciños, P., Vázquez, J. A., \& Pandiella, S. S. (2013). Inhibition kinetics of lipid oxidation of model foods by using antioxidant extract of fermented soybeans. Food Chemistry, 139(1-4), 837-844. 